\title{
Robust Tracking Commitment
}

\author{
Altuğ Bitlislioğlu, Tomasz T. Gorecki, Colin N. Jones
}

\begin{abstract}
Many engineering problems that involve hierarchical control applications, such as demand side ancillary service provision to the power grid, can be posed as a robust tracking commitment problem. In this setting, the lower-level controller commits a set of possible reference trajectories over a finite horizon to an external entity in exchange for a reward corresponding to the size of the reference set and the allowed margin of tracking error. If the commitment is accepted, the lower-level system is required to track any reference trajectory that can be sampled from the committed set. This paper presents the framework of robust tracking commitment and a method to solve the optimal commitment problem for constrained linear systems subject to uncertain disturbance and reference signals. The proposed method allows tractable computations via convex optimization for conic representable uncertainty sets and lends itself to distributed solution methods. We demonstrate the proposed method in a simulation based case study with a commercial building that offers frequency regulation service to the power grid.
\end{abstract}

Index Terms-tracking, hierarchical control, distributed control, robust control, service commitment, ancillary service, demand response

\section{INTRODUCTION}

$\mathbf{R}$ EGULATION of large and complex systems that include many agents is usually handled with several control layers that interact in a hierarchical fashion in order to break down the complex control task into simpler sub-tasks. Frequency regulation of the power grid is a good example of such a setting, where an upper level controller, run by the grid operator, sends reference signals to the lower level subsystems, reserve providers, which are expected to track the reference within an acceptable error bound. For the upper level controller, it is crucial to know the tracking capability of the subsystems. In the power grid, this information is provided to the grid operator in terms of a reserve commitment, which represents the set of robustly trackable power generation/consumption trajectories by the lower level subsystem for a specific time window [1]. The assessment of the optimal reserve capacity of a large scale complex subsystem, such as a commercial building, is far from trivial. The difficulty comes from large dimensional models that limits available computational methods and the fact that the controlled system is restrained by its primary objectives and external disturbances such as weather conditions and occupancy levels.

In this paper, motivated by the reserve commitment problem for the power grid, we formulate the robust tracking commitment problem for constrained linear systems. The problem corresponds to the assessment of a subsystem's tracking capability for a finite horizon and optimal commitment of this capability to an upper control layer. Of course, the subsystem's overall

The authors are with the Laboratoire d'Automatique, École Polytechnique Fédérale de Lausanne (EPFL), Lausanne, Switzerland (e-mail: altug.bitlislioglu@epfl.ch, tomasz.gorecki@epfl.ch, colin.jones@epfl.ch ). cost of operation and feasibility have to be taken into account in this assessment. The tracking capability can be expressed in terms of a set of possible reference trajectories the system can track robustly. By robustness, we mean that the subsystem will not violate internal constraints and always stay close to the revealed reference trajectory within an allowed error set, while being subjected to external disturbances.

For a fixed reference set over a finite horizon, considering that both the reference and disturbance are external uncertainty sources, the ability of the system to track any reference from this set can be assessed by means of a standard robust optimization problem [2], [3], given that the system is linear, constraint and uncertainty sets are polytopic. The peculiarity of the commitment problem comes from the freedom in the choice of uncertainty sets that are admissible for robust tracking, since it is up to the subsystem to determine the size of uncertainty set it can handle. In other words, we are faced with a robust optimization problem, in which the size of the uncertainty set is also a decision variable.

Finite horizon robust control for linear systems is well established in the model predictive control (MPC) literature [4]. However, the related work is mainly concerned with obtaining a control policy that guarantees robust feasibility and stability under a given uncertainty set. Available methods mainly rely on choosing a nominal trajectory and a control policy that will keep the system around the nominal trajectory under the effect of the uncertainty $[5]-[8]$. The use of a closed-loop control policy reduces the conservatism significantly compared to open-loop robust policies.

Among many available robust MPC methods, we build on the affine disturbance feedback method [8] which is a translation of the work of [9] to the MPC framework. The method can be directly applied to conic representable uncertainty sets over the whole prediction horizon, capturing the temporal correlation of the uncertain signal while eliminating the need of enumerating extreme trajectories. Affine disturbance feedback policies are shown to be equivalent to time-varying state feedback policies [4], [8] and the method allows online optimization of the policy via convex optimization, rather than working with an a priori fixed policy. Other tube MPC methods that can supersede the disturbance feedback approach [6], [7], rely heavily on the assumption of uncorrelated realization of the uncertainty at each time step. This assumption restricts the family of trajectory sets that can be used to characterize the inherent tracking capability of the system. In our work, we take into account the temporal correlation of the uncertainty, especially for the reference signal, and hence consider a more expressive family of sets for characterizing the inherent capability of the system. We demonstrate the advantage of this approach in the simulation study in Section $\mathrm{V}$. Considering temporal correlation for uncertainty modeling is also found 
out to be beneficial in the context of the multistage economic dispatch problem [10]. In addition, the machinery of affine, or equivalently linear, policies for treatment of uncertainty can be also used to obtain more generic nonlinear policies [11]. The work of [11] generalizes linear policies by considering lifted uncertainty sets under a nonlinear operator, a possibility also shown by [9]. In Section IV-B, we show that this enhancement of linear policies can be incorporated in the robust tracking commitment problem in a computationally tractable manner, whenever the nature of the uncertainty set allows for it.

An illustrative example of robust model predictive control applied to the operation of the power grid can be found in [12], where the authors allocate reserves while considering temporal correlation of the demand-generation forecast and assuming the forecast error to belong to a polytopic set defined over a finite prediction horizon. However, the uncertainty set is fixed prior to allocation, therefore the authors do not address the problem of assessing disturbance rejection capabilities of a given reserve fleet. The commitment problem, on the other hand, requires searching over uncertainty sets that the system can accommodate rather than guaranteeing robustness against a fixed uncertainty set. The interest in this problem has peaked in recent years due to the prospect of demand response applications [13], where the reference set to be tracked that represents the reserve capacity of the system is not given, but should be computed by the service provider. In [3] the problem is posed in the robust model predictive control context, and simple up-down flexibility of a single actuator is optimized. [14] and [15] consider aggregation of several subsystems to track a reference signal and optimize maximum up-down limits on the reference, however the robust formulation is again limited either to single dedicated actuators or predetermined schemes that distribute the required change in the total power consumption among actuators. [16] considers reference sets that are norm balls and optimizes over linear mappings to modify the uncertain reference set utilizing dual norm formulations. However, in all aforementioned works on demand response, the authors do not consider the temporal correlation that will be present in the uncertain reference signal. This problem is tackled in our previous work [2], where we consider polytopic uncertainty sets defined over the whole prediction horizon and formulate a method for modification of the uncertainty set via linear maps. A similar work is [17], where the authors propose optimizing over a linear map to be applied to a polytopic reference set that represents energy constraints in frequency regulation signals.

Other works have tackled similar problems in more specific contexts. [18] and [19] propose aggregation methods for characterizing the power consumption flexibility of a collection of thermostatically controlled loads (TCLs). This can be considered as a particular case of the commitment problem and can be addressed with the methodology proposed in this paper, that can also handle more general systems. Another related work is [20] where the problem of serving a set of time constrained load requests (such as electric vehicle charging) is tackled. Sufficient conditions are given for a supply profile to be able to serve the loads and a dispatch strategy is proposed. This work differs from ours by considering a continuous time formulation and again using the specific structure of the problem to derive a solution.

A connected problem is the output regulation, which deals with the capability of the system to track a reference trajectory that is generated by an external dynamical system [21]. In the finite horizon framework, the external system serves as a generator for the reference trajectory set. Most of the work in output regulation deals with asymptotic tracking guarantees [21], [22]. Similarly, for systems under additive disturbance, the authors of [23] show robust convergence to a neighborhood of a fixed reference that is allowed to change occasionally. However, within the finite horizon tracking commitment context, it is necessary to guarantee tracking during the whole commitment period, which includes transients. Therefore, similar to [24], we consider tracking requirements during the whole horizon, including the transients, rather than showing convergence to a neighborhood of a constant reference. In this direction, the authors of [25] utilize robust invariant sets to guarantee tracking with specified error bounds during and after the finite prediction horizon. The guarantees are sought for a given reference generator under the assumption that there exists a feasible solution to the problem. However, none of the aforementioned works in guaranteed tracking or output regulation framework consider the problem of modulating the reference set while solving the control problem.

The idea of modulating initial sets for inner approximation of admissible sets is used in [6]. Namely the authors develop a method to partially design control policies online, by using approximations of state and input tubes, which overcomes the difficulty of designing fixed local controllers in the regular tube MPC [5]. Even though both the framework and the methodology are significantly different than our case, we take a standpoint similar to that of [6], in the sense of applying modifier functions to fixed shaped simple sets for recovering inner approximations of more complex sets.

The robust commitment problem can be classified as a generalized semi-infinite program [26] due to the variability of the uncertainty set. This kind of problems is in general more difficult to solve than semi-infinite programs, that is robust optimization problems with a fixed uncertainty set [27]. The related literature is mostly concerned with generic nonlinear programs and global optimization methods, that can be computationally demanding $[27]-[30]$. Within the framework of linear systems and polytopic state and input constraints, we reformulate the robust tracking commitment problem such that it can be approximated by a simpler robust optimization problem. The novelty of our approach comes from the notion of modifier functions that are used to modulate uncertainty sets, which enables reformulation of the commitment problem into a standard multistage adjustable robust program that can be solved efficiently [9]. The solution method is based on linear decision rules [8], [9] together with linear modifier functions over conic representable sets and can be used for sizing of reference sets while simultaneously guaranteeing robust tracking.

The results represented in this paper are extensions of our previous work [2]. Here, we formulate a general ro- 
bust tracking commitment problem for linear systems that allows practical computation of optimal reference sets to be committed for guaranteed tracking. The contributions are the formulation of the robust tracking commitment framework and enhancement of similar formulations presented in [2], [16], [17] in three ways. First we incorporate generic output tracking, where the output is a function of both input and the state of the system, rather than just the input. Second we consider additional disturbances acting on the system as another source of uncertainty and allow for inexact tracking with certified error bounds. Finally, we establish sufficient conditions for modifier functions and control policies on uncertainty sets, that ensure causal admissibility for robust tracking according to the available information on the uncertainty.

The paper is organized as follows. Section II lays out the problem formulation for constrained tracking under uncertainty and introduces information structures. Section III defines the robust tracking commitment problem, and discusses sufficient conditions for admissibility of uncertainty sets and control policies. Section IV presents a method for solving the problem in a computationally tractable manner with implicit modulation of uncertainty sets and closes with discussions of properties of the tractable problem formulation. Finally, Section $\mathrm{V}$ illustrates our results with a demand response application.

Notation: $\mathbb{R}^{n}$ denotes the Euclidean space of dimension $n$, and $\mathbb{Z}$ denotes the set of integers. For two integers $i \in \mathbb{Z}$ and $j \in \mathbb{Z}$ such that $i<j$, let $\mathbb{Z}_{[i, j]}:=\{i, i+1, \ldots, j\}$. $I_{n}$ denotes the identity matrix of dimension $n$ and $\otimes$ denotes the Kronecker product. For a matrix $\mathbf{M} \in \mathbb{R}^{n \times m}$, an integer $i \in \mathbb{Z}_{[1, n]}$ and a set $\mathcal{J} \subseteq \mathbb{Z}_{[1, m]}, \mathbf{M}(i, \mathcal{J})$ indicates the set of components that belong to the $i$ th row and columns for which the indices belong to $\mathcal{J}$. For a set $\mathcal{Q} \subseteq \mathbb{R}^{n} \times \mathbb{R}^{m}$, the orthogonal projection operator is defined as $\operatorname{Proj}_{x}(\mathcal{Q}):=\left\{x \in \mathbb{R}^{n} \mid \exists y \in \mathbb{R}^{m},(x, y) \in \mathcal{Q}\right\}$. Given two functions $f: \mathbb{R}^{n} \rightarrow \mathbb{R}^{m}$ and $g: \mathbb{R}^{m} \rightarrow \mathbb{R}^{l}, f \circ g: \mathbb{R}^{n} \rightarrow \mathbb{R}^{l}$ denotes the composition of $f$ and $g$, such that $f \circ g(x)=$ $f(g(x))$.

\section{PROBLEM FORMULATION}

\section{A. Constrained tracking under uncertainty}

Consider the linear uncertain system

$$
\begin{aligned}
x_{k+1} & =A x_{k}+B u_{k}+w_{k} \\
y_{k} & =C x_{k}+D u_{k}
\end{aligned}
$$

with constrained state and inputs $(x, u) \in X \times U \subset \mathbb{R}^{n_{x}} \times \mathbb{R}^{n_{u}}$, disturbance $w \in \mathbb{R}^{n_{x}}$ and output $y \in \mathbb{R}^{n_{y}}$. The sets $X$ and $U$ are assumed to be convex, polytopic and compact.

Given that the system is in state $x$ at time 0 , the input sequence $\boldsymbol{u}=\left(u_{0}, \ldots, u_{N-1}\right)$ is applied, and the disturbance sequence $\boldsymbol{w}=\left(w_{0}, \ldots, w_{N-1}\right)$ is observed, the state at time $i$ is denoted by $\phi_{i}(x, \boldsymbol{u}, \boldsymbol{w})$, and the resulting sequence of states $\left(\phi_{1}(x, \boldsymbol{u}, \boldsymbol{w}), \ldots, \phi_{N}(x, \boldsymbol{u}, \boldsymbol{w})\right)$ by $\boldsymbol{\phi}(x, \boldsymbol{u}, \boldsymbol{w})$. Note that $\boldsymbol{\phi}$ is a linear function of its arguments. The output of the system over the horizon is also a linear function, and is denoted by $\boldsymbol{y}(x, \boldsymbol{u}, \boldsymbol{w}):=\left(C x_{0}+D u_{0}, C \phi_{1}+D u_{1}, \ldots, C \phi_{N-1}+\right.$ $\left.D u_{N-1}\right)$, where the arguments have been removed for brevity.
Given a reference signal $r \in \mathbb{R}^{n_{y}}$ at time $k$, the tracking error is denoted by $e_{k}=y_{k}-r_{k}$, whereas for a reference sequence $\boldsymbol{r}:=\left(r_{0}, \ldots, r_{N-1}\right)$ the corresponding error sequence over the finite horizon is given by

$$
\boldsymbol{e}:=\boldsymbol{r}-\boldsymbol{y}(x, \boldsymbol{u}, \boldsymbol{w}) .
$$

In order to qualify tracking, we introduce the tracking error set $\mathcal{E} \subset \mathbb{R}^{N n_{y}}$. The objective of the control action is to maintain the difference between the output of the system and the reference signal, that is $e$, within the set $\mathcal{E}$.

We can now define the set of input, reference and disturbance sequences that satisfy the system and tracking constraints over an $N$-step horizon

$$
\mathcal{Q}(x):=\{(\boldsymbol{u}, \boldsymbol{r}, \boldsymbol{w}) \mid \boldsymbol{\phi}(x, \boldsymbol{u}, \boldsymbol{w}) \in \mathcal{X}, \boldsymbol{u} \in \mathcal{U}, \boldsymbol{e} \in \mathcal{E}\}
$$

where $\mathcal{X}:=X^{N}$ and $\mathcal{U}:=U^{N}$. As seen from the definition, the feasibility set $\mathcal{Q}$ is parameterized by the initial condition $x$ of the system. In the following, we will drop the argument of $\mathcal{Q}$ for notational simplicity.

Remark 1. The reference is only defined over the finite horizon and we do not discuss tracking guarantees for the infinite horizon case. Note that any terminal condition that would ensure robust invariance under a persistent disturbance after the finite horizon can easily be added to the set $\mathcal{Q}(x)$ without changing its structure. At this point, we acknowledge the difficulty of constructing invariant sets for self-correlated or state dependent uncertainties [31]. Therefore we consider tracking obligation only over the finite horizon, and advise using standard robustly invariant sets generated for an uncorrelated disturbance after the finite horizon. The reader is referred to [32] for computation of positively robust invariant sets under time invariant disturbance sets, to be used as the terminal constraint.

Our goal is to determine if, starting from a given initial state $x$, an admissible control policy exists such that the output $y$ of system (1) can robustly track a reference signal $r$, that is all system and tracking constraints can be satisfied, for all possible realizations of the reference sequence $r$ and disturbance sequence $\boldsymbol{w}$ such that

$$
\boldsymbol{r} \in \mathcal{R}, \quad \boldsymbol{w} \in \mathcal{W}
$$

where $\mathcal{R} \subset \mathbb{R}^{N n_{y}}$ and $\mathcal{W} \subset \mathbb{R}^{N n_{x}}$ represent the reference set and the disturbance set and are assumed to be compact. If such a control policy exists, then we can conclude that the system is qualified for robust tracking with respect to the bounded sets $\mathcal{R}$ and $\mathcal{W}$. Note that this definition of the reference and disturbance sets over entire sequences allows temporal correlation along the horizon, providing a critical flexibility that will be exploited in the application studied in Section V.

Both the reference $\boldsymbol{r}$ and disturbance $\boldsymbol{w}$ are exogenous uncertain signals for the system. From the point of view of the controller, the main difference between these two is the time they are observed by the controller. This causality condition can be easily incorporated in the control policy to be applied over the horizon as we will show in the following 
sections. Therefore, we define $\boldsymbol{\xi}=(\boldsymbol{r}, \boldsymbol{w}) \in \mathbb{R}^{n_{\xi}}$ as the unified uncertain signal. We further define the general uncertainty set as

$$
\Xi=\mathcal{R} \times \mathcal{W}
$$

Let the map $\pi_{k}: \mathbb{R}^{N n_{\xi}} \rightarrow \mathbb{R}^{n_{u}}$ be the control policy to be used at time step $k$. The control policy sequence for the finite horizon can be defined as

$$
\boldsymbol{\pi}(\boldsymbol{\xi})=\left(\pi_{0}(\boldsymbol{\xi}), \ldots, \pi_{N-1}(\boldsymbol{\xi})\right)
$$

We can now define the set of all admissible finite-horizon control policies mapping from disturbance and reference sequences to input sequences

$$
\Delta(\Xi):=\{\boldsymbol{\pi}: \Xi \rightarrow \mathbb{U} \mid \forall \boldsymbol{\xi} \in \Xi,(\boldsymbol{\pi}(\boldsymbol{\xi}), \boldsymbol{\xi}) \in \mathcal{Q}\}
$$

Given the feasibility and tracking conditions and the uncertainty set, a controller using a policy that belongs to the set $\Delta(\Xi)$, starting from the state $x$, can keep the tracking error within the set $\mathcal{E}$ throughout the finite horizon for any realization of the disturbance $\boldsymbol{w}$ and the reference $r$. The existence of such control policies is not guaranteed: if the system constraints and the tracking set is too restrictive or the uncertainty sets are too large, then it may not be possible for any controller to satisfy system feasibility and tracking requirements simultaneously.

Let us now characterize the uncertainty sets that allow existence of admissible control policies for tracking. This characterization will be instrumental in the following sections, when we optimize over reference sets that the system can track robustly.

Definition 1. The set $\Xi \subset \mathbb{R}^{N n_{\xi}}$ is admissible for tracking by system (1) in state $x$ if

$$
\Delta(\Xi) \neq \emptyset
$$

The following lemma provides more insight into the geometry of the admissibility condition:

Lemma 1. The set $\Xi \subset \mathbb{R}^{N n_{\xi}}$ is admissible for tracking by system (1) in state $x$ if and only if:

$$
\Xi \subseteq \operatorname{Proj}_{\boldsymbol{\xi}}(\mathcal{Q})
$$

where $\operatorname{Proj}_{\xi}(\mathcal{Q})$ denotes the projection of the set $\mathcal{Q}$ onto the $\xi$ subspace.

Proof. : The proof directly follows from the definition of the projection operator and the definition of $\Delta(\Xi)$. Suppose that $\Xi \subseteq \operatorname{Proj}_{\boldsymbol{\xi}}(\mathcal{Q})$, we have that $\forall \boldsymbol{\xi} \in \Xi, \exists \boldsymbol{u}:(\boldsymbol{u}, \boldsymbol{\xi}) \in \mathcal{Q}$. This indicates the existence of a function $\pi$, which maps every $\boldsymbol{\xi} \in \Xi$ to a feasible $\boldsymbol{u}=\boldsymbol{\pi}(\boldsymbol{\xi})$, such that $(\boldsymbol{\pi}(\boldsymbol{\xi}), \boldsymbol{\xi}) \in \mathcal{Q}$, and hence $\Delta(\Xi) \neq \varnothing$. Conversely, suppose that $\Delta(\Xi) \neq \varnothing$ and let $\boldsymbol{\pi} \in \Delta(\Xi)$. By definition of $\Delta(\Xi), \forall \boldsymbol{\xi} \in \Xi, \exists \boldsymbol{u}$ such that $\boldsymbol{u}=\boldsymbol{\pi}(\boldsymbol{\xi})$ and $(\boldsymbol{u}, \boldsymbol{\xi}) \in \mathcal{Q}$, and hence $\Xi \subseteq \operatorname{Proj}_{\boldsymbol{\xi}}(\mathcal{Q})$.

Lemma 1 also illuminates a method of testing the admissibility of a given uncertainty set for robust tracking, by means of verifying set inclusion.

\section{B. Information structure of control policies}

The control policy $\pi$ should account for the fact that the uncertain exogenous signals are revealed partially to the controller as time progresses. Generally speaking, any decision variable $u_{k}$ might depend on a subset of the uncertainty vector $\xi$ and only on this subset. To make this claim more precise, the concept of the information structure of a function $f$ is introduced. The presentation follows concepts from Section 14.2 of [9] but adopts a different formulation.

Definition 2. Let $\mathcal{I}$ be a subset of $\{1,2, \ldots, n\}$, and

$$
\mathcal{F}(\mathcal{I})=\left\{f: \mathbb{R}^{n} \rightarrow \mathbb{R} \mid x_{\mathcal{I}}=\hat{x}_{\mathcal{I}} \Rightarrow f(x)=f(\hat{x})\right\}
$$

where $x_{\mathcal{I}}$ denotes the entries of $x$ defined by the indices of $\mathcal{I}$. Let $\mathcal{I}=\left(\mathcal{I}_{k}\right)_{k \in \mathbb{Z}_{[1, m]}}$ be a collection of index subsets and

$$
\mathcal{F}(\mathcal{I})=\left\{f: \mathbb{R}^{n} \rightarrow \mathbb{R}^{m}, f_{k} \in \mathcal{F}\left(\mathcal{I}_{k}\right) \forall k \in \mathbb{Z}_{[1, m]}\right\}
$$

If $f \in \mathcal{F}(\mathcal{I})$, then we refer to $\mathcal{I}$ as the information structure of $f$.

Loosely speaking, $\mathcal{F}(\mathcal{I})$ denotes the set of real-valued functions that depend only on the input indexed in $\mathcal{I}$. For functions with multiple outputs, the information structure is defined output-wise. $\mathcal{I}$ summarizes the information structure of the function $f$ : the $k^{\text {th }}$ component of $f$ depends only on inputs indexed in $\mathcal{I}_{k}$. For example, in the robust multistage control setting considered here, a typical requirement of the control policy will be causality (also called nonanticipativity) which states that the current control action can depend on observations made in the past; in our notation, this fact translates to: for each stage, every control action can depend on past measurements, so that $\pi_{k} \in \mathcal{F}\left(\mathcal{I}_{k}\right)$ with $\mathcal{I}_{k}=\{1, \ldots, k-1\}$. Notice here a small abuse of notation in the sense that $\pi_{k}$ is a function with values in $\mathbb{R}^{n_{u}}$, and by $\pi_{k} \in \mathcal{F}\left(\mathcal{I}_{k}\right)$ we mean that every component of $\pi_{k}$ is in $\mathcal{F}\left(\mathcal{I}_{k}\right)$.

Definition 3. The set $\Xi \subset \mathbb{R}^{N n_{\xi}}$ is causally admissible for tracking by system (1) in state $x$ with respect to the information structure $\mathcal{I}$ if

$$
\mathcal{F}(\mathcal{I}) \cap \Delta(\Xi) \neq \emptyset .
$$

In contrast to Definition 1, we now require that the control policy satisfies a particular information structure. For example, the reference trajectory to track will usually be known at the current time step but not the disturbance. It is also possible that the reference is known either partially or totally in advance. In Section III] we will see that our examples may display more complex information structures.

\section{Robust Tracking Commitment}

Consider the problem of finding a reference set, such that the combined uncertainty set $\Xi=\mathcal{R} \times \mathcal{W}$, composed of the disturbance and reference, is admissible for robust tracking for system (1). The admissible reference set $\mathcal{R}$ is to be committed to an external agent together with the guarantee of robust tracking for a finite horizon. We call this problem, a robust tracking commitment problem, the application of which can be found in ancillary service provision to the power grid, as we 
will demonstrate in Section V] or similar hierarchical control settings.

Essentially, tracking commitment requires evaluation of the admissibility of uncertainty sets, in order to be able to find a suitable reference set to be committed. Based on Definition 3 , we can write the family of causally admissible uncertainty sets for tracking with respect to a given information structure

$$
\Omega=\left\{\Xi \subset \mathbb{R}^{N n_{\xi}} \mid \exists \boldsymbol{\pi} \in \mathcal{F}(\mathcal{I}) \cap \Delta(\Xi)\right\}
$$

First we tackle the problem of simply finding a causally admissible reference set, without attaching any cost function to the problem. The tracking commitment problem can be written as

$$
\text { find } \mathcal{R}: \mathcal{R} \times \mathcal{W} \in \Omega
$$

For a fixed uncertainty set, admissibility can be verified by searching over control policies. However it is not obvious how to search over possible admissible sets and corresponding control policies simultaneously. In order to treat the problem with a unified methodology, we will characterize admissible sets as images of a modifier function applied to an initial uncertainty set $\hat{\Xi}$. The advantage of this approach will be evident in the following section IV, when we formulate computationally tractable methods for evaluating the admissibility of uncertainty sets for tracking, utilizing parameterized function based techniques available in the robust optimization literature [9].

\section{A. Implicit modification of uncertainty sets}

Let us formalize characterization of uncertainty sets by modifier functions. We first define the uncertainty modifier function $\nu: \mathbb{R}^{N n_{\xi}} \rightarrow \mathbb{R}^{N n_{\xi}}$, which is assumed to be bijective and used for reshaping a given uncertainty set.

$$
\boldsymbol{\nu}(\hat{\Xi})=\{\boldsymbol{\nu}(\boldsymbol{\xi}): \boldsymbol{\xi} \in \hat{\Xi}\}
$$

Remark 2. Note that we do not distinguish between the reference and the disturbance as they are just parts of the combined uncertainty signal, and all presented results apply to general robust programs. However, in the context of tracking commitment, only the reference set $\mathcal{R}$ can be modulated, whereas the disturbance set $\mathcal{W}$ is fixed. Also in general, the information structure of the reference and the disturbance are different. Nevertheless, without loss of generality, these particularities of the uncertain signal can be easily incorporated in the definition of the information structure and the mapping $\nu$ by letting

$$
\boldsymbol{\nu}(\boldsymbol{\xi})=\boldsymbol{\nu}(\boldsymbol{r}, \boldsymbol{w}):=\left(\boldsymbol{\nu}_{r}(\boldsymbol{r}), \boldsymbol{w}\right) .
$$

In the following, we will show that we can evaluate the admissibility of the set $\Xi=\boldsymbol{\nu}(\hat{\Xi})$ via conditions on the composite function $\hat{\boldsymbol{\pi}}=\boldsymbol{\pi} \circ \boldsymbol{\nu}$ that is applied to the initial set $\hat{\Xi}$, as depicted in Figure 1. This allows us to fix an initial uncertainty set $\hat{\Xi}$, embed the modifier function into the control policy and implicitly modulate uncertainty sets and control policies simultaneously. To this end we introduce the following lemma,
Lemma 2. Let $\boldsymbol{\nu}: \mathbb{R}^{N n_{\xi}} \rightarrow \mathbb{R}^{N n_{\xi}}$, be a bijection and $\hat{\Xi}$ be a compact set with non-empty interior. The set $\Xi:=\boldsymbol{\nu}(\hat{\Xi})$ is causally admissible for tracking by system (1) in state $x$ with respect to the information structure $\mathcal{I}$ if and only if

$$
\exists \hat{\boldsymbol{\pi}} \in \Delta_{\boldsymbol{\nu}}(\hat{\Xi}): \hat{\boldsymbol{\pi}} \circ \nu^{-1} \in \mathcal{F}(\mathcal{I})
$$

where $\Delta_{\nu}$ is defined as

$$
\Delta_{\nu}(\Xi):=\{\boldsymbol{\pi}: \forall \boldsymbol{\xi} \in \Xi,(\boldsymbol{\pi}(\boldsymbol{\xi}), \boldsymbol{\nu}(\boldsymbol{\xi})) \in \mathcal{Q}\}
$$

Proof. : Suppose $\hat{\boldsymbol{\pi}} \in \Delta_{\boldsymbol{\nu}}(\hat{\Xi})$ and $\hat{\boldsymbol{\pi}} \circ \boldsymbol{\nu}^{-1} \in \mathcal{F}(\mathcal{I})$. Then we have

$$
\forall \hat{\boldsymbol{\xi}} \in \hat{\Xi},(\hat{\boldsymbol{\pi}}(\hat{\boldsymbol{\xi}}), \boldsymbol{\nu}(\hat{\boldsymbol{\xi}})) \in \mathcal{Q}
$$

Let $\boldsymbol{\xi}:=\boldsymbol{\nu}(\hat{\boldsymbol{\xi}})$. Since $\boldsymbol{\nu}$ is bijective, we have $\hat{\boldsymbol{\xi}}=\boldsymbol{\nu}^{-1}(\boldsymbol{\xi})$. Therefore (18) is equivalent to

$$
\begin{aligned}
& \forall \boldsymbol{\nu}^{-1}(\boldsymbol{\xi}) \in \hat{\Xi},\left(\hat{\boldsymbol{\pi}} \circ \boldsymbol{\nu}^{-1}(\boldsymbol{\xi}), \boldsymbol{\nu} \circ \boldsymbol{\nu}^{-1}(\boldsymbol{\xi})\right) \in \mathcal{Q} \\
\Leftrightarrow & \forall \boldsymbol{\xi} \in \boldsymbol{\nu}(\hat{\Xi}),\left(\hat{\boldsymbol{\pi}} \circ \boldsymbol{\nu}^{-1}(\boldsymbol{\xi}), \boldsymbol{\xi}\right) \in \mathcal{Q} \\
\Leftrightarrow & \forall \boldsymbol{\xi} \in \Xi,(\boldsymbol{\pi}(\boldsymbol{\xi}), \boldsymbol{\xi}) \in \mathcal{Q} \text { with } \boldsymbol{\pi}=\hat{\boldsymbol{\pi}} \circ \boldsymbol{\nu}^{-1} \\
\Leftrightarrow & \boldsymbol{\pi} \in \Delta(\Xi)
\end{aligned}
$$

Moreover, we have that $\boldsymbol{\pi}=\hat{\boldsymbol{\pi}} \circ \boldsymbol{\nu}^{-1} \in \mathcal{F}(\mathcal{I})$. This concludes that $\Xi$ is causally admissible for tracking according to Definition 3. The reverse direction follows from the equivalence of all steps.

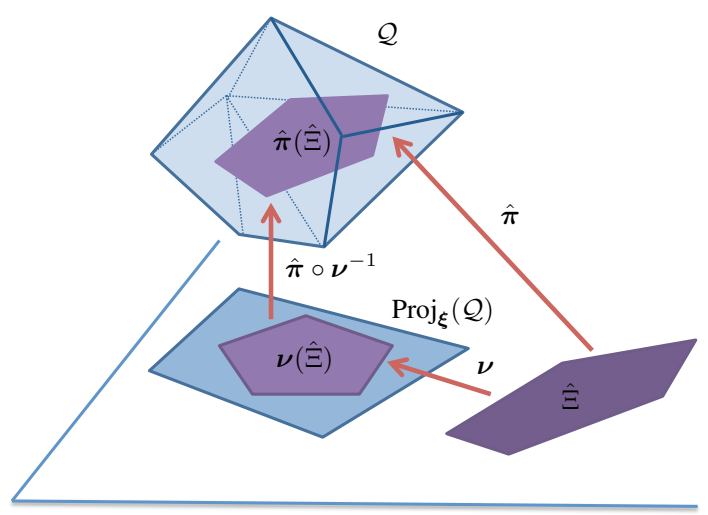

Fig. 1: Conceptual sketch of the relationships between uncertainty sets and applied functions. The initial uncertainty set $\hat{\Xi}$ is not necessarily a subset of the projection of $\mathcal{Q}$, therefore might not be admissible according to Lemma 1 However once we find a feasible lifting of this set into $\mathcal{Q}$, we can take its projection as an admissible uncertainty set, which is given by $\Xi=\boldsymbol{\nu}(\hat{\Xi})$. The corresponding admissible control policy can be obtained by letting $\boldsymbol{\pi}=\hat{\boldsymbol{\pi}} \circ \boldsymbol{\nu}^{-1}$.

Remark 3. Since the modifier function $\boldsymbol{\nu}$ is an arbitrary bijection, we do not lose generality when we consider uncertainty sets that can be characterized as the image of a given initial compact set $\hat{\Xi}$ with non-empty interior, under $\nu$. 
According to Lemma 2 we can write an equivalent formulation of the family of admissible sets for a given initial set $\hat{\Xi}$

$$
\Omega=\left\{\begin{array}{l|l}
\Xi \subset \mathbb{R}^{N n_{\xi}} \mid \begin{array}{l}
\exists \boldsymbol{\nu}, \hat{\boldsymbol{\pi}}: \Xi=\boldsymbol{\nu}(\hat{\Xi}), \\
\hat{\boldsymbol{\pi}}: \in \Delta_{\boldsymbol{\nu}}(\hat{\Xi}), \hat{\boldsymbol{\pi}} \circ \nu^{-1} \in \mathcal{F}(\mathcal{I})
\end{array}
\end{array}\right\}
$$

When we look for a causally admissible set that belongs to $\Omega$, the description (20) allows us to implicitly manipulate uncertainty sets and control policies simultaneously to verify admissibility, as will be seen in Section IV] However, while searching for a modifier function $\nu$, the condition $\hat{\boldsymbol{\pi}} \circ \boldsymbol{\nu}^{-1} \in \mathcal{F}(\mathcal{I})$ is difficult to evaluate since it is a condition on a composite function that involves the inverse of $\nu^{-1}$. In the following, we will propose a simple sufficient condition directly on $\nu$, that is easy to evaluate and ensures causal admissibility of the modified uncertainty set. We start by splitting the causality conditions of the composite function $\hat{\boldsymbol{\pi}} \circ \boldsymbol{\nu}^{-1}$.

Lemma 3. Let $\left(\mathcal{I}_{k}\right)_{k \in \mathbb{Z}_{[1, m]}}$ be a set of information structures and $f: \mathbb{R}^{n} \longrightarrow \mathbb{R}$. If for all $k, f \in \mathcal{F}\left(\mathcal{I}_{k}\right)$ then $f \in \mathcal{F}\left(\bigcap_{k} \mathcal{I}_{k}\right)$.

The proof of Lemma 3 , as well as other technical proofs in this section are grouped in Appendix B The results will be briefly discussed in this section and the reader is referred to Appendix $B$ for more details. Lemma 3 states an intuitive fact, that is if the output of a function $f$ depends only on inputs indexed by $\mathcal{I}_{1}$ and $\mathcal{I}_{2}$, then it actually depends only on inputs indexed by their intersection. This directly motivates the next lemma.

Lemma 4. Let $\boldsymbol{g}: \mathbb{R}^{n} \rightarrow \mathbb{R}^{n}$, be a bijection. Given an information structure $\mathcal{I}$, define $\hat{\mathcal{I}}$ as

$$
\hat{\mathcal{I}}_{j}=\bigcap_{\left\{i \mid j \in \mathcal{I}_{i}\right\}} \mathcal{I}_{i}
$$

The following equivalence holds

$$
\forall \boldsymbol{f} \in \mathcal{F}(\mathcal{I}), \boldsymbol{f} \circ \boldsymbol{g} \in \mathcal{F}(\mathcal{I}) \Longleftrightarrow \boldsymbol{g} \in \mathcal{F}(\hat{\mathcal{I}})
$$

Equation 21) characterizes a set of functions which do not change the information structure of $\boldsymbol{f}$. Loosely speaking, it states that if $\boldsymbol{f}_{i}$ depends on $x_{j}$ then $\boldsymbol{g}_{j}$ should not depend on anything that $\boldsymbol{f}_{i}$ does not depend on. Notice that $\hat{\mathcal{I}}_{j}$ is always nonempty and in particular it contains $j$. This reflects the fact that a "diagonal" mapping (where $\boldsymbol{g}_{j}$ depends only on $j$ for all $j$ ) does not change the information structure of any function it is composed with (for linear functions it means that multiplying by a diagonal matrix always preserves the sparsity pattern).

In Figure 2, the information structure $\hat{\mathcal{I}}$ for different information structures $\mathcal{I}$ are presented. The $k^{\text {th }}$ row of the matrix represents the indicator vector of $\mathcal{I}_{k}$. These matrices can be thought of as sparsity patterns in the case that the control policies are linear. The first column shows the sparsity pattern of the control policy $\pi$ and the second column the corresponding sparsity-preserving sparsity pattern. In other words, multiplying the matrix from the first column by the

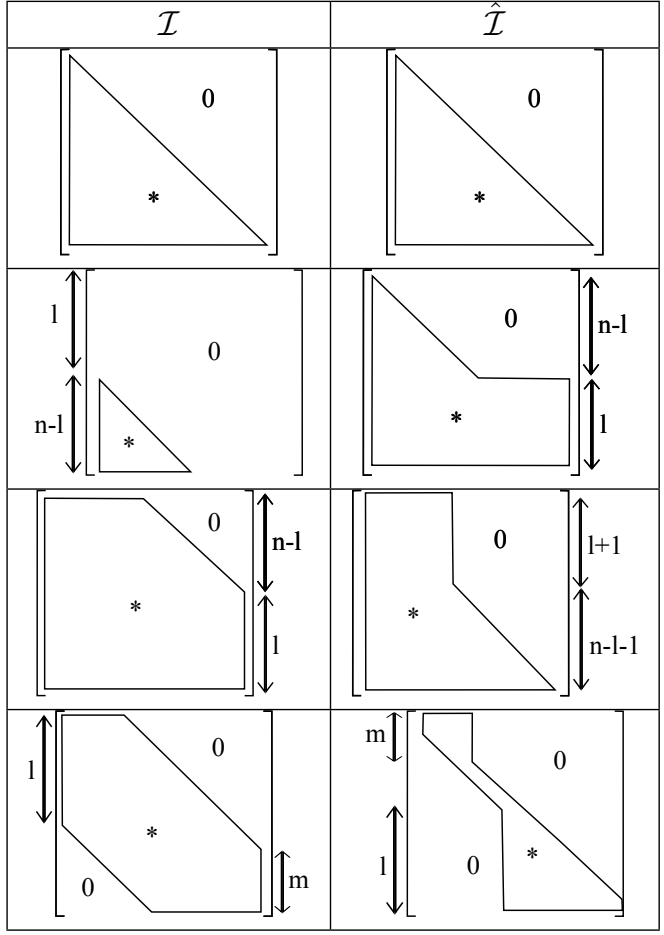

Fig. 2: For given information structures, the corresponding information structure of the uncertainty modifier function

matrix from the second column will result in the same sparsity pattern. This directly helps us select control policies and modifier functions such that their composition will still respect the required information structure. For example, as would be expected, the first row of Figure 2 tells us that a lower triangular control policy composed with a lower triangular modifier will still be lower triangular. However more complex features in $\mathcal{I}$, such as delays and forecasting, result in nontrivial sparsity patterns for $\hat{\mathcal{I}}$.

\section{B. Sufficient conditions for causal admissibility of modified uncertainty sets}

In view of Lemma 2, simultaneous optimization over $\pi$ and $\boldsymbol{\nu}$ would be beneficial for searching admissible uncertainty sets. Lemma 4 is instrumental in proving that from a control policy $\hat{\boldsymbol{\pi}}$ defined on $\hat{\Xi}$ and an invertible mapping $\boldsymbol{\nu}$, a control policy defined on $\boldsymbol{\nu}(\hat{\Xi})$ which has the desired information structure can be recovered. Indeed, $\hat{\boldsymbol{\pi}} \in \mathcal{F}(\mathcal{I})$ and $\boldsymbol{\nu}^{-1} \in \mathcal{F}(\hat{\mathcal{I}})$ ensures that $\hat{\boldsymbol{\pi}} \circ \boldsymbol{\nu}^{-1}$ defined on $\boldsymbol{\nu}(\hat{\Xi})$ belongs to $\mathcal{F}(\mathcal{I})$ according to the lemma.

However, conditions on $\nu^{-1}$ are inconvenient since the aim is to optimize directly over $\boldsymbol{\nu}$. Sufficient conditions on $\boldsymbol{\nu}$ are sought to replace the condition $\nu^{-1} \in \mathcal{F}(\hat{\mathcal{I}})$. Unfortunately a certain information structure for $\nu^{-1}$ does not usually result in a specific information structure for $\nu$. In particular, a sparse information structure for $\nu^{-1}$ does not generally result in a sparse information structure for $\boldsymbol{\nu}$. For example, the inverse of a causal function is not generally causal. The following lemma gives sufficient conditions on $\boldsymbol{\nu}$. 
Lemma 5. Suppose $\boldsymbol{\nu}: \mathbb{R}^{n} \longrightarrow \mathbb{R}^{n}$ is a continuous bijection of $\mathbb{R}^{n}$ and $\boldsymbol{\nu} \in \mathcal{F}(\hat{\mathcal{I}})$ as defined by equation 21]. Define $\mathcal{G}=\{\boldsymbol{f} \circ \boldsymbol{\nu} \mid \boldsymbol{f} \in \mathcal{F}(\mathcal{I})\}$. We have

$$
\mathcal{G}=\mathcal{F}(\mathcal{I})
$$

Under mild assumptions, Lemma 5 states that composing $f \in \mathcal{F}(\mathcal{I})$ with $\nu$ results in a function with the same information structure.

Corollary 1. Given an information structure $\mathcal{I}$ and $\hat{\mathcal{I}}$ as defined in equation (21), if $\boldsymbol{\nu}$ is a continuous bijection and $\boldsymbol{\nu} \in \mathcal{F}(\hat{\mathcal{I}})$, then $\boldsymbol{\nu}^{-1} \in \mathcal{F}(\hat{\mathcal{I}})$.

Proof. We have $\mathcal{F}(\mathcal{I})=\{\boldsymbol{f} \mid \boldsymbol{f} \in \mathcal{F}(\mathcal{I})\}=\{\boldsymbol{f} \circ \boldsymbol{\nu} \circ$ $\left.\nu^{-1} \mid f \in \mathcal{F}(\mathcal{I})\right\}$ by bijectivity of $\boldsymbol{\nu}$. In turn, $\mathcal{F}(\mathcal{I})=$ $\left\{\boldsymbol{g} \circ \boldsymbol{\nu}^{-1} \mid \boldsymbol{g} \in \mathcal{G}\right\}$ by definition of $\mathcal{G}$. By Lemma 5 , using the assumptions on $\boldsymbol{\nu}$, we have that $\mathcal{G}=\mathcal{F}(\mathcal{I})$ and therefore $\mathcal{F}(\mathcal{I})=\left\{\boldsymbol{g} \circ \boldsymbol{\nu}^{-1} \mid \boldsymbol{g} \in \mathcal{F}(\mathcal{I})\right\}$. Hence, for any $\boldsymbol{g} \in \mathcal{F}(\mathcal{I})$, it holds that $\boldsymbol{g} \circ \boldsymbol{\nu}^{-1} \in \mathcal{F}(\mathcal{I})$. The fact that $\boldsymbol{\nu}^{-1} \in \mathcal{F}(\hat{\mathcal{I}})$ then follows from Lemma 4

Theorem 1. Let $\boldsymbol{\nu}: \mathbb{R}^{N n_{\xi}} \rightarrow \mathbb{R}^{N n_{\xi}}$, be a continuous bijection and $\mathcal{I}$ an information structure, $\hat{\mathcal{I}}$ defined by equation (21) and $\Delta_{\nu}$ in equation (17). $\nu(\hat{\Xi})$ is causally admissible for tracking with respect to the information structure $\mathcal{I}$ if

$$
\begin{aligned}
& \mathcal{F}(\mathcal{I}) \cap \Delta_{\nu}(\hat{\Xi}) \neq \emptyset \\
& \nu \in \mathcal{F}(\hat{\mathcal{I}})
\end{aligned}
$$

Proof. Suppose there exists $\hat{\pi} \in \mathcal{F}(\mathcal{I}) \cap \Delta_{\nu}(\hat{\Xi})$. Since $\nu$ is a continuous bijection, $\boldsymbol{\nu} \in \mathcal{F}(\hat{\mathcal{I}})$ implies that $\boldsymbol{\nu}^{-1} \in \mathcal{F}(\hat{\mathcal{I}})$ by Corollary 1 Lemma 4 in turn ensures that $\hat{\boldsymbol{\pi}} \circ \boldsymbol{\nu}^{-1} \in \mathcal{F}(\mathcal{I})$. Finally, application of Lemma 2 concludes the proof.

Theorem 1 provides sufficient conditions for causal admissibility of an uncertainty set for tracking. We can define the family of admissible sets that comply with these sufficient conditions as

$$
\tilde{\Omega}(\hat{\Xi})=\left\{\begin{array}{l|l}
\Xi \subset \mathbb{R}^{N n_{\xi}} & \begin{array}{l}
\exists \boldsymbol{\nu}, \hat{\boldsymbol{\pi}} \\
\Xi=\boldsymbol{\nu}(\hat{\Xi}), \nu \in \mathcal{F}(\hat{\mathcal{I}}) \\
\hat{\boldsymbol{\pi}} \in \mathcal{F}(\mathcal{I}) \cap \Delta_{\nu}(\hat{\Xi})
\end{array}
\end{array}\right\}
$$

For the definition of $\tilde{\Omega}$ we have replaced the condition $\hat{\boldsymbol{\pi}} \circ \boldsymbol{\nu}^{-1} \in \mathcal{F}(\mathcal{I})$ with the sufficient but simpler conditions $\hat{\boldsymbol{\pi}} \in \mathcal{F}(\mathcal{I})$ and $\boldsymbol{\nu} \in \mathcal{F}(\hat{\mathcal{I}})$. Therefore $\tilde{\Omega}$ is a restriction of the original family of admissible sets $\Omega$.

$$
\tilde{\Omega}(\hat{\Xi}) \subseteq \Omega
$$

The restriction will depend on the initial set $\hat{\Xi}$ and thus the argument of $\tilde{\Omega}$ is added to reflect this fact. However, this restriction leads to tractable formulations based on the available robust programming literature, as we will show in the next section.

Finally, we write the modified robust tracking commitment problem that is based on sufficient conditions (23) as

$$
\text { find } \mathcal{R}: \mathcal{R} \times \mathcal{W} \in \tilde{\Omega}(\hat{\Xi})
$$

\section{TRACTABLE APPROXIMATIONS}

The problem formulation (26) allows us to search over uncertainty sets implicitly by means of modifier functions. However the problem is still difficult in its general form, due to the infinite dimension of the search space and the infinite number of constraints. Therefore we will look for finite dimensional and tractable approximations of the tracking commitment problem in order to solve it efficiently.

Using the definitions of $\tilde{\Omega}$ and $\Delta_{\nu}$, we can rewrite the modified robust tracking commitment problem as

$$
\begin{array}{ll}
\text { find } & \hat{\boldsymbol{\pi}}, \boldsymbol{\nu} \\
\text { subject to } & \forall \hat{\boldsymbol{\xi}} \in \hat{\Xi} \\
& (\hat{\boldsymbol{\pi}}(\hat{\boldsymbol{\xi}}), \boldsymbol{\nu}(\hat{\boldsymbol{\xi}})) \in \mathcal{Q} \\
& \hat{\boldsymbol{\pi}} \in \mathcal{F}(\mathcal{I}) \\
& \boldsymbol{\nu} \in \mathcal{F}(\hat{\mathcal{I}}) .
\end{array}
$$

Note that 27) is an adjustable robust optimization (ARO) problem [9], that allows decisions to be taken after the realization of the uncertainty. In our case the uncertainty dependent decision rule is characterized by the control policy $\pi$. In the standard form of ARO, the uncertainty set is fixed, whereas the tracking commitment problem requires optimization over possible uncertainty sets. However, even with the additional modification of the uncertainty set, the tracking commitment problem (27) still fits into the standard ARO framework, because the uncertainty modifier $\boldsymbol{\nu}$ can also be treated as a decision rule. Consequently, we can directly utilize results from adjustable robust programming [9]. However, tractable adjustable robust programming methods presented in [9] is applicable only to robust linear optimization problems. This means that the constraints of the optimization problem that is affected by the uncertainty can only be described by linear inequalities. In order to satisfy this criterion on the problem structure, we rely on the following assumption.

Assumption 1. The system in consideration is linear and described by (1). State and inputs of the system are subject to polytopic constraints. The allowed error set $\mathcal{E}$ is polytopic.

Following Assumption 1] we define the polytopic tracking error set as

$$
\mathcal{E}:=\{e \mid G e \leqslant g\}
$$

The feasibility set $\mathcal{Q}$ also becomes polytopic and can be written as

$$
\mathcal{Q}=\{(\boldsymbol{u}, \boldsymbol{\xi}) \mid H \boldsymbol{u}+Q \boldsymbol{\xi} \leqslant q\}
$$

For the derivation of $Q, H$, and $q$ see Appendix A Note that $q$ is an affine function of the initial condition $x$, and $g$, which determines the size of the tracking set. This polytopic description of the feasibility set allows treatment of the modified robust tracking commitment problem (27) in the uncertain linear optimization framework.

Until this point, we have not made any strong assumptions on the families of uncertainty sets, policy and modifier functions. The presented theory in section IIII applies to generic functions and sets. Therefore, the sufficient conditions in (24) 
can be used to verify causal admissibility of any uncertainty set, using generic policies and modifier functions. In the following we restrict ourselves to sets for which computationally tractable methods can be derived for verification of admissibility conditions (24).

Consider the feasibility condition in 27 with polytopic $\mathcal{Q}$. Ensuring satisfaction of constraints under the worst case realization of the uncertainty leads to a set of inner maximization problems.

$$
\max _{\hat{\boldsymbol{\xi}} \in \hat{\Xi}}\{H \hat{\boldsymbol{\pi}}(\hat{\boldsymbol{\xi}})+Q \boldsymbol{\nu}(\hat{\boldsymbol{\xi}})\} \leqslant q
$$

where the max operator applies row-wise. The authors of [9] show that if the max problem is a convex conic linear program (LP), one can replace the maximisation with its dual minimization problem. Thereafter, it is not necessary to solve the min problem, since existence of a feasible dual variable will ensure that the max in $(28)$ is less than $q$. Therefore the original semiinfinite constraint under uncertainty can be transformed into a finite dimensional constraint on the dual variables. We omit the details of the method for brevity and focus on the application of these results in our framework. The reader is referred to [9] for details and [33] for a tutorial review.

Following the results of [9], in order to be able to obtain tractable robust counterpart formulations, we introduce the following assumption.

Assumption 2. The uncertainty sets under consideration are representable by intersections of convex cones as

$$
\hat{\Xi}=\left\{\boldsymbol{\xi} \mid F_{i} \boldsymbol{\xi}+f_{i} \in \mathbf{K}_{i}, i \in \mathbb{Z}_{[1, m]}\right\}
$$

where the cone $\mathbf{K}_{i}$ is proper.

Note that, the considered class of uncertainty sets is very extensive, as it allows the description of well known cones such as the non-negative orthant, the Lorentz cone and the positive semi-definite cone as well as their intersections and products.

In the following, we present the tractable approximations of (27) using linear and nonlinear policy and modifier functions.

\section{A. Linear policy and modifier functions}

In this section we build our formulation on the results of [9] which shows that restricting the search space of policies to linear (or affine) functions leads to finite dimensional and tractable formulations of adjustable robust programs, referred as the affinely adjustable robust counterpart (AARC). For notational simplicity, we use linear functions, as any affine term that does not depend on the realization of the uncertainty can be defined as a separate non-adjustable decision variable in the optimization problem.

Let us define the linear versions of the control policy and the uncertainty modifier

$$
\boldsymbol{\pi}_{l i n}(\boldsymbol{\xi}):=\mathbf{M} \boldsymbol{\xi}, \quad \boldsymbol{\nu}_{l i n}(\boldsymbol{\xi})=\mathbf{L} \boldsymbol{\xi}
$$

where $\mathbf{M} \in \mathbb{R}^{N n_{u} \times N n_{\xi}}$ and $\mathbf{L} \in \mathbb{R}^{N n_{\xi} \times N n_{\xi}}$. We can describe the causality conditions by constraints on $\mathbf{M}$ and $\mathbf{L}$

$$
\begin{array}{ll}
\mathbf{M}\left(k, \mathbb{Z}_{\left[1, N n_{\xi}\right]} \backslash \mathcal{I}_{k}\right)=0, \quad k \in \mathbb{Z}_{[1, N]} & \Leftrightarrow \boldsymbol{\pi} \in \mathcal{F}(\mathcal{I}) \\
\mathbf{L}\left(k, \mathbb{Z}_{\left[1, N n_{\xi}\right]} \backslash \hat{\mathcal{I}}_{k}\right)=0, \quad k \in \mathbb{Z}_{[1, N]} & \Leftrightarrow \boldsymbol{\nu} \in \mathcal{F}(\hat{\mathcal{I}})
\end{array}
$$

Note that the constraints (31) impose that the elements of $\mathbf{M}$ and $\mathbf{L}$ multiplying the elements of the uncertain variable which are not included in the information structure at step $k$ to be zero, thus enforcing causality of the linear functions $\boldsymbol{\pi}_{l i n}$ and $\boldsymbol{\nu}_{\text {lin }}$.

Let us now formulate the robust tracking commitment problem (27) with linear policies given in 30 and conic uncertainty sets described by 29

$$
\begin{array}{ll}
\text { find } & \hat{\mathbf{M}}, \mathbf{L} \\
\text { subject to } & \forall \hat{\boldsymbol{\xi}}: F_{i} \hat{\boldsymbol{\xi}}+f_{i} \in \mathbf{K}_{i}, \quad i \in \mathbb{Z}_{[1, m]} \\
& H \hat{\mathbf{M}} \hat{\boldsymbol{\xi}}+Q \mathbf{L} \hat{\boldsymbol{\xi}} \leqslant q \\
& (\hat{\mathbf{M}}, \mathbf{L}) \text { satisfies 31 }
\end{array}
$$

Once the problem is solved, a feasible solution $\hat{\mathbf{M}}^{*}$ and $\mathbf{L}^{*}$ can be used to construct the uncertainty set that is causally admissible for tracking and the corresponding control policy

$$
\Xi=\mathbf{L}^{*} \hat{\Xi}, \quad \boldsymbol{\pi}(\boldsymbol{\xi})=\mathbf{M} \boldsymbol{\xi}, \quad \mathbf{M}=\hat{\mathbf{M}}^{*} \mathbf{L}^{*-1}
$$

To recover a tractable formulation of (32), the worst case realizations of the uncertainty can be considered by enforcing the constraint; $\max _{\hat{\boldsymbol{\xi}} \in \hat{\Xi}}\{H \mathbf{M} \hat{\boldsymbol{\xi}}+Q \mathbf{L} \hat{\boldsymbol{\xi}}\} \leqslant q$. Applying conic duality thereafter and stacking dual variables in matrix $\mathbf{Z}$, the robust counterpart can be formulated as

$$
\begin{array}{ll}
\text { find } & \mathbf{Z}, \hat{\mathbf{M}}, \mathbf{L} \\
\text { subject to } & \mathbf{Z}_{i}^{T} \in \mathbf{K}_{i}^{*}, i \in \mathbb{Z}_{[1, m]} \\
& \sum_{i=1}^{m} \mathbf{Z}_{i} f_{i} \leqslant q \\
& \sum_{i=1}^{m} \mathbf{Z}_{i} F_{i}=-(H \hat{\mathbf{M}}+Q \mathbf{L})
\end{array}
$$

$(\hat{\mathbf{M}}, \mathbf{L})$ satisfies 31

The reader is referred to [9] for the derivation. The robust counterpart for the tracking commitment problem (32) is convex in linear control policies parameterized by $\hat{\mathbf{M}}$ and linear uncertainty modifiers parameterized by $\mathbf{L}$. Therefore, when sets $\mathbf{K}_{i}$ are polyhedral, second order or semi-definite cones, the problem formulation (34) allows tractable computations of feasible reference sets admissible for tracking with respect to the information structure $\mathcal{I}$ and the tracking error set $\mathcal{E}$, by system (1). Table I gives a summary of problem complexity in case of most common uncertainty sets for the reference and disturbance.

Remark 4. Any additional decision variable that is independent of the uncertainty, $\overline{\boldsymbol{u}}$, as well as non zero nominal 
values for the disturbance and reference, $\overline{\boldsymbol{\xi}}$, can be easily incorporated in the above formulation as

$$
H \overline{\boldsymbol{u}}+Q \overline{\boldsymbol{\xi}}+\sum_{i=1}^{m} \mathbf{Z}_{i} f_{i} \leqslant q
$$

\begin{tabular}{cc|c}
$\mathcal{R}$ & $\mathcal{W}$ & Robust Counterpart \\
\hline$F_{r} \boldsymbol{r} \leqslant f_{r}$ & $F_{w} \boldsymbol{w} \leqslant f_{w}$ & LP \\
$F_{r} \boldsymbol{r}+f_{r}:\|\boldsymbol{r}\|_{2} \leqslant 1$ & $F_{w} \boldsymbol{w} \leqslant f_{w}$ & SOCP \\
$F_{r} \boldsymbol{r} \leqslant f_{r}$ & $F_{w} \boldsymbol{w}+f_{w}:\|\boldsymbol{w}\|_{2} \leqslant 1$ & SOCP \\
$F_{r} \boldsymbol{r}+f_{r}:\|\boldsymbol{r}\|_{2} \leqslant 1$ & $F_{w} \boldsymbol{w}+f_{w}:\|\boldsymbol{w}\|_{2} \leqslant 1$ & SOCP
\end{tabular}

TABLE I: Optimization type for the robust counterpart formulation (34) of the tracking commitment problem, considering combinations of polytopes and ellipsoids as uncertainty sets. Note that the polytopic representation also covers 1 and $\infty$ norm balls. LP stands for linear program and SOCP stands for second-order cone program.

\section{B. Nonlinear policy and uncertainty modifiers}

The formulation (34) is restricted to linear functions, but in certain cases it is possible to deal with nonlinear policies (or modifier functions) in a computationally tractable manner. The key principle, introduced in [9] and studied in greater detail in [11] is to consider a modified uncertainty set which is the image of the original uncertainty under a nonlinear lifting. If the lifted uncertainty set or its convex hull can be represented in the conic form of 29), the machinery of linear policies and modifier functions can be applied. The combination of the nonlinear lifting and linear policy and modifiers results in nonlinear policy and modifiers.

Consider again the constraints:

$$
H \boldsymbol{\pi}(\boldsymbol{\xi})+Q \boldsymbol{\nu}(\boldsymbol{\xi}) \leqslant q, \quad \forall \boldsymbol{\xi} \in \Xi
$$

We define the lifted uncertainty variable, and the corresponding uncertainty set as

$$
\mathcal{Z}:=\{\boldsymbol{\zeta}=\Lambda(\boldsymbol{\xi}), \mid \boldsymbol{\xi} \in \Xi\}
$$

with $\Lambda: \mathbb{R}^{k} \longrightarrow \mathbb{R}^{k^{\prime}}$ a nonlinear lifting operator. Following assumptions of [11], we may assume that there exists a retraction operator $\rho$ such that $\rho \circ \Lambda=\mathbb{I}_{k}$, the identity operator. This implies that $k^{\prime} \geq k$ and $\Lambda$ is injective. We can now choose the policy and modifier functions under the form:

$$
\boldsymbol{\pi}(\boldsymbol{\xi})=\tilde{\mathbf{M}} \boldsymbol{\zeta}=\tilde{\mathbf{M}} \Lambda(\boldsymbol{\xi}) \text { and } \boldsymbol{\nu}(\boldsymbol{\xi})=\tilde{\mathbf{L}} \boldsymbol{\zeta}=\tilde{\mathbf{L}} \Lambda(\boldsymbol{\xi})
$$

and the objective is then to find $\tilde{\mathbf{M}}$ and $\tilde{\mathbf{L}}$ such that:

$$
H \tilde{\mathbf{M}} \boldsymbol{\zeta}+Q \tilde{\mathbf{L}} \boldsymbol{\zeta} \leqslant q, \quad \forall \boldsymbol{\zeta} \in \operatorname{conv}(\mathcal{Z})
$$

If the new extended uncertainty set $\mathcal{Z}$ or its convex hull $\operatorname{conv}(\mathcal{Z})$, can be represented in conic form (29), then the tractable formulation (34) can be used to solve this problem. As a result, a feasible solution $\tilde{\mathbf{M}}^{*}$ and $\tilde{\mathbf{L}}^{*}$ can be used to construct the uncertainty set that is causally admissible for tracking and the corresponding control policy:

$$
\Xi=\tilde{\mathbf{L}}^{*} \mathcal{Z}, \quad \boldsymbol{\pi}(\boldsymbol{\xi})=\mathbf{M} \boldsymbol{\xi}, \quad \mathbf{M}=\tilde{\mathbf{M}}^{*}\left(\tilde{\mathbf{L}}^{*} \Lambda\right)^{-1}
$$

To ensure that $\left(\tilde{\mathbf{L}}^{*} \Lambda\right)$ is invertible, a simple solution is for example to take $\tilde{\mathbf{L}}$ under the form $\tilde{\mathbf{L}}=\mathbf{L} \circ \rho$ with $\mathbf{L}$ invertible.
The causality of the resulting policies can be ensured by considering the information structure for the lifted uncertainty $\zeta$ and applying requirement 31.

A list of tractable cases has been identified in the robust programming literature including quadratic policies for ellipsoidal uncertainty sets, piecewise linear continuous policies with box uncertainty sets, polynomial policies with box uncertainty sets. The reader is referred to [9] and [11] for more details. Note that other works such as [34] propose mixed-integer reformulations for other types of lifting operators. As an example, we briefly summarize results from [9] showing that quadratic control policies can be handled with ellipsoidal uncertainty sets. Consider the ellipsoidal uncertainty set:

$$
\Xi=\left\{\boldsymbol{\xi} \mid\|\mathcal{T} \boldsymbol{\xi}\|_{2} \leqslant 1\right\}
$$

with invertible $\mathcal{T}$. The lifted uncertainty is:

$$
\boldsymbol{\zeta}=\left(1, \boldsymbol{\xi},\left\{\boldsymbol{\xi}_{i} \boldsymbol{\xi}_{j}, \forall i, j\right\}\right)
$$

Or using a matrix notation:

$$
\boldsymbol{\zeta}=\left[\begin{array}{cc}
1 & \xi^{T} \\
\boldsymbol{\xi} & \boldsymbol{\xi} \xi^{T}
\end{array}\right]
$$

As shown in [9], the convex hull of the lifted uncertainty set $\mathcal{Z}$ is given by

$$
\operatorname{conv}(\mathcal{Z})=\left\{\boldsymbol{\zeta}=\left[\begin{array}{ll}
1 & \boldsymbol{\xi}^{T} \\
\boldsymbol{\xi} & W
\end{array}\right] \mid \zeta \succeq 0, \operatorname{tr}\left(\mathcal{T} W \mathcal{T}^{T}\right) \leqslant 1\right\}
$$

where $\zeta \succeq 0$ means that $\zeta$ is a symmetric positive semidefinite matrix. This representation can be put in the standard conic form of (29), and therefore allows a tractable robust counterpart formulation like (34). Notice that a linear policy in $\zeta$ is equivalent to a quadratic policy in the $\boldsymbol{\xi}$ of the form:

$$
\boldsymbol{\pi}_{i}(\boldsymbol{\xi}):=\boldsymbol{\xi}^{T} Y_{i} \boldsymbol{\xi}+v_{i}^{T} \boldsymbol{\xi}+u_{i}
$$

As an application example, in [35], the authors use quadratic liftings to find the largest volume inner approximations of polytope projections. For our purposes, evaluation of nonlinear policies for robust tracking commitment is subject to ongoing research.

\section{Modulating the tracking error set}

In Sections IV-A and IV-B, we have formulated a tractable version of the robust tracking commitment problem (13) which seeks a causally admissible reference set with respect to a fixed tracking error set $\mathcal{E}$. However for some applications, it might be preferable to modulate the reference set together with the associated tracking error set, since the relative sizes of the two sets will indicate the tracking performance. For example, for frequency regulation service to the power grid in Switzerland, the service providers are allowed to deviate from the reference up to a certain percentage of the total service capacity committed, therefore a service provider who is committing a larger reference set is allowed to have a larger error set [36].

Notice that the problem (34) is convex in $q$ which parameterizes the polytopic feasibility set $\mathcal{Q}$. Therefore one can freely optimize over modifications of the feasibility set. For clarity 
let us write the system feasibility and tracking constraints separately as

$$
\mathcal{Q}=\left\{\begin{array}{l|l}
(\boldsymbol{u}, \boldsymbol{r}, \boldsymbol{w}) & \begin{array}{l}
T \boldsymbol{u}+V \boldsymbol{w} \leqslant h \\
G(\boldsymbol{r}-(P \boldsymbol{u}+S \boldsymbol{w}+\overline{\boldsymbol{y}})) \leqslant g
\end{array}
\end{array}\right\}
$$

where $\overline{\boldsymbol{y}}$ is the nominal output of the system without control action and the derivation of matrices $T, V, P, S$ can be found in Appendix A

From (45), we can immediately observe that the problem (34) is also convex in $g$ which parameterizes the tracking error set $\mathcal{E}$, therefore allows modulation in a tractable manner. The sizes of the tracking error set and the reference set can be related by enforcing a joint constraint on the uncertainty modifier function parameter $\mathbf{L}$ and the error set parameter $g$.

\section{Optimal tracking commitment}

As mentioned earlier, the robust tracking commitment (13) is a feasibility problem. On the other hand, the optimal robust tracking commitment problem is finding the control policy, reference and tracking error sets, that minimize a cost function.

$$
\underset{\pi \in \mathcal{F}(\mathcal{I}) \cap \Delta(\mathcal{R} \times \mathcal{W})}{\operatorname{minimize}} J(\boldsymbol{\pi}, \mathcal{R}, \mathcal{E})
$$

Relying on the tractable formulation with linear control policies and uncertainty modifiers (32), we can solve the tractable version of the optimal robust tracking commitment.

$$
\begin{array}{ll}
\operatorname{minimize} & J\left(\boldsymbol{u}, \mathbf{L}_{r}, g\right) \\
\text { subject to } & \forall \hat{\boldsymbol{r}}: F_{r} \hat{\boldsymbol{r}}+f_{r} \in \mathbf{K}_{r}, \\
& \forall \boldsymbol{w}: F_{r} \boldsymbol{w}+f_{w} \in \mathbf{K}_{w}, \\
& T \boldsymbol{u}+V \boldsymbol{w} \leqslant h \\
& G\left(\mathbf{L}_{r} \hat{\boldsymbol{r}}-(P \boldsymbol{u}+S \boldsymbol{w}+\overline{\boldsymbol{y}})\right) \leqslant g \\
& \boldsymbol{u}=\hat{\mathbf{M}}_{r} \hat{\boldsymbol{r}}+\mathbf{M}_{w} \boldsymbol{w}+\overline{\boldsymbol{u}}
\end{array}
$$

$(\hat{\mathbf{M}}, \mathbf{L})$ satisfies 31

where $\hat{\mathbf{M}}$ and $\mathbf{L}$ are defined as

$$
\hat{\mathbf{M}}=\left[\begin{array}{ll}
\hat{\mathbf{M}}_{r} & \\
& \mathbf{M}_{w}
\end{array}\right], \quad \mathbf{L}=\left[\begin{array}{ll}
\mathbf{L}_{r} & \\
& \mathbf{I}_{N n_{x}}
\end{array}\right]
$$

For notational simplicity, the reference and disturbance sets are represented as single conic sets, but they can also be defined as intersection of several conic sets as in (29).

With a suitable cost function, the optimal commitment problem 47) can be solved as explained in section IV. The cost function usually depends on the uncertain realization of the reference and disturbance. However this dependence can be qualified out by either considering the worst-case or expectation of the possible cost realizations [37]. Furthermore, as long as $J$ is bilinear in the uncertain variables and decision variables, the tractable robust formulation for the minimization of an upper bound, that constitutes an equivalent problem with certain cost function, can be obtained [9].

After solving (47), the optimal control policy, reference and tracking error sets can be obtained as

$$
\begin{aligned}
& \mathcal{R}^{*}=\left\{\boldsymbol{r}=\mathbf{L}_{r}^{*} \hat{\mathbf{r}} \mid F_{r} \hat{\boldsymbol{r}}+f_{r} \in \mathbf{K}_{r}\right\} \\
& \boldsymbol{\pi}^{*}(\boldsymbol{r}, \boldsymbol{w})=\hat{\mathbf{M}}_{r}^{*} \mathbf{L}_{r}^{*-1} \boldsymbol{r}+\mathbf{M}_{w}^{*} \boldsymbol{w}+\overline{\boldsymbol{u}}^{*} \\
& \mathcal{E}^{*}=\left\{\boldsymbol{e} \mid G \boldsymbol{e} \leqslant g^{*}\right\}
\end{aligned}
$$

where ${ }^{*}$ indicates that the variable is an optimizer of (47).

\section{E. Collective tracking}

In this section, we will consider the collective tracking commitment problem, where the reference signal is to be tracked by the summation (or average) of the outputs of several subsystems that are not coupled via constraints or dynamics. In this case, the aggregate tracking error can be written as

$$
\boldsymbol{e}=\boldsymbol{r}-\sum_{j=1}^{n} \boldsymbol{y}^{j}
$$

where superscript $j$ indicates that the associated variable belongs to subsystem $j$.

In order to compute a causally admissible reference set for the collection of subsystems, it is necessary to compute the aggregated tracking capability simultaneously. An obvious option is to treat the collective system as a single system with block diagonal system matrices, and solve the problem 32, centrally. However this requires collecting the knowledge of detailed subsystem models by an aggregator and will possibly result in a very large number of decision variables. Therefore it is desirable to distribute the problem, such that it can be solved without central knowledge of the models and using limited communication between the agents.

Let us briefly show that the problem (32) in fact easily lends itself to distributed solution methods. The subsystems are supposed to track a single reference by collective action. This is equivalent to saying that the subsystems are tracking separate reference signals, which sum up to the central reference. Using a common nominal reference set and a linear uncertainty modifier function for each subsystem, the reference can be split as

$$
\boldsymbol{r}^{j}=\mathbf{L}^{j} \hat{\boldsymbol{r}}, \quad \sum_{j=1}^{n} \mathbf{L}^{j}=\mathbf{L}
$$

where L parameterizes the global linear modifier function. Therefore the aggregate reference set can be described as

$$
\mathcal{R}=\sum_{j=1}^{n} \mathbf{L}^{j} \hat{\mathcal{R}}
$$

Many distribution schemes are possible, given the costreward framework of the collective tracking task. As an example, we consider the case where the error set is fixed, the reward is split between agents according to their contributions to tracking characterized by parameter $\mathbf{L}^{j}$ and the objective is to minimize the total cost

$$
\sum_{j=1}^{n} J^{j}\left(\boldsymbol{\pi}^{j}, \mathbf{L}^{j}\right)
$$

Subsystem feasibility constraints $T^{j} \boldsymbol{u}^{j}+V^{j} \boldsymbol{w}^{j} \leqslant h^{j}$ and causality conditions on the local control policies can be treated separately by each subsystem. However (51) and the collective tracking constraint [50) introduces a constraint coupling 
among all subsystems. The collective tracking constraint can be written as

$$
\begin{aligned}
& \forall \hat{\boldsymbol{r}}: F_{r} \hat{\boldsymbol{r}}+f_{r} \in \mathbf{K}_{r}, \quad \forall \boldsymbol{w}^{j}: F_{w}^{j} \boldsymbol{w}^{j}+f_{w}^{j} \in \mathbf{K}_{w}^{j} \\
& \sum_{j=1}^{n}\left(G\left(\mathbf{L}^{j} \hat{\boldsymbol{r}}-\left(P^{j} \boldsymbol{u}^{j}+S^{j} \boldsymbol{w}^{j}+\overline{\boldsymbol{y}}^{j}\right)\right)\right) \leqslant g \\
& \boldsymbol{u}^{j}=\hat{\mathbf{M}}_{r}^{j} \hat{\boldsymbol{r}}+\mathbf{M}_{w}^{j} \boldsymbol{w}^{j}+\overline{\boldsymbol{u}}^{j}
\end{aligned}
$$

Utilizing linear control policies $\boldsymbol{u}^{j}=\hat{\mathbf{M}}_{r}^{j} \hat{\boldsymbol{r}}+\mathbf{M}_{w}^{j} \boldsymbol{w}^{j}+\overline{\boldsymbol{u}}^{j}$ and formulating the dual of the robust counterpart as in (34), we obtain a tractable formulation for the collective tracking constraint

$$
\begin{array}{ll}
\mathbf{Z}_{r}^{T} \in \mathbf{K}_{r}^{*}, \quad \mathbf{Z}_{w}^{j}{ }^{T} \in \mathbf{K}^{j^{*}}, & \\
\mathbf{Z}_{r} f_{r}+\sum_{j=1}^{n}\left(\mathbf{Z}_{w}^{j} f_{w}^{j}-P^{j} \overline{\boldsymbol{u}}^{j}-\overline{\boldsymbol{y}}^{j}\right) \leqslant g \in \mathbb{Z}_{[1, n]} \\
\mathbf{Z}_{r} F_{r}=\sum_{j=1}^{n} G\left(P^{j} \hat{\mathbf{M}}_{r}^{j}-\mathbf{L}^{j}\right), \\
\mathbf{Z}_{w}^{j} F_{w}^{j}=G\left(P^{j} \mathbf{M}_{w}^{j}+S^{j}\right), &
\end{array}
$$

We observe that the coupling constraints are 55b and (55c). The partial Lagrangian with the coupling constraints can be written as

$$
\begin{gathered}
\sum_{j=1}^{n} J^{j}\left(\pi^{j}, \mathbf{L}^{j}\right)+\lambda^{T} \operatorname{vec}\left(\mathbf{Z}_{r} F_{r}-\sum_{j=1}^{n} G\left(P^{j} \hat{\mathbf{M}}_{r}^{j}-\mathbf{L}^{j}\right)\right) \\
+\mu^{T}\left(\mathbf{Z}_{r} f_{r}+\sum_{j=1}^{n}\left(\mathbf{Z}_{w}^{j} f_{w}^{j}-P^{j} \overline{\boldsymbol{u}}^{j}-\overline{\boldsymbol{y}}^{j}\right)-g\right)
\end{gathered}
$$

which is separable given the variables $\lambda, \mu$ and $\mathbf{Z}_{r}$. Therefore the collective tracking commitment problem can be solved in a distributed manner with global updates of these variables or enforcing consensus on their local copies [38]. Note that, after the commitment of the aggregate reference set, it is not necessary to further communicate for guaranteed tracking if all subsystems have access to the aggregate reference $\boldsymbol{r}$, since collective tracking is robustly guaranteed by independent local control policies. However, the performance can be improved by repetitively solving the problem (55) online for redistributing the tracking task among subsystems according to the available information on the disturbance and reference as time progresses. Adjusting the error set parameter $g$ is also possible with a suitable constraint on the aggregate modifier $\mathbf{L}$ and $g$. In case of dynamic couplings, inter-system constraints or common disturbances that couples the state and inputs of several subsystems, the same methodology can be applied to derive the tractable robust counterpart formulation and distribute the computation.

\section{APPLICATIONS}

In this section, we will illustrate most of the theoretical concepts put forward in this article on a realistic example of a building providing ancillary services to the power grid. This application requires a simple characterization of the tracking capabilities of a building, such that the power system operator can coordinate many service providers. The same methodology can be applied to similar multi-agent frameworks such as coordination of drones [39], considering feasible trajectories that each drone can follow. Characterization of workspaces of machine tools and feasible torque references of engines in hybrid cars can be listed as other possible applications [25].

\section{A. Power tracking with a building}

The problem of interest here is the commitment of secondary frequency control provision. Secondary frequency control providers in the Swiss electricity market offer regulation power with a weekly bid. To do so, they first have to schedule their baseline power consumption ahead of time over the contract period by purchasing energy on day ahead or intraday markets. Secondly, they have to evaluate how much deviation around this baseline they can accommodate. For that they have to provide one number $\lambda$ called the capacity bid that represents the maximum positive or negative power consumption deviation they are willing to support over the period of the contract. For example, if $\lambda=10 \mathrm{~kW}$, it means they theoretically could be required to increase (or decrease) their power consumption by $10 \mathrm{~kW}$ for the period of commitment. In practice they receive a signal from the grid operator, called Area Control Signal (ACS), within the bounds specified by the bids. At the time they receive this signal, the building controller needs to make sure its power consumption is the sum of the baseline consumption purchased and the ACS. Deviations in the power consumption tracking are allowed within an error margin proportional to the bid. For details on frequency control, the reader is referred to [40].

Notice that the robust tracking method proposed in this paper is particularly suited for this application. Indeed, all possible values of the ACS can be represented by a reference set $\mathcal{R}$, that the building operator can modify (essentially in this case, scale up or down depending on the value of $\lambda$ ). An optimal set size can be computed provided that an appropriate cost function is chosen as will be demonstrated next. By choosing $y$ defined in equation (1) to be the total power consumption of the building and $\mathcal{X}$ and $\mathcal{U}$ to represent the operating constraints of the building, we can see that the methodology proposed in this paper directly allows to address this problem.

We consider an office building with three controlled zones served by individual air handling units that we assume can control the heat fluxes to the zones. A linear state-space model of the building was extracted and validated against EnergyPlus simulation data using the toolbox OpenBuild [41]. The toolbox builds a thermal model of the building based on first principle modeling and collects realistic data for occupancy and equipment schedules, as well as weather. One week of typical summer weather for the city of Chicago is used in this study. The model of the building is a model of the form (1) with state dimension $n_{x}=10$ and input dimension $n_{u}=3$. The disturbance captures the effect of internal gains, solar radiations and outdoor temperature, and the input vector represents the thermal power input power to the zones (which is negative since it is cooling season). In this study, $y$ is a 
scalar that represents the total electricity consumption so that $y_{k}=\alpha \sum_{i=1}^{n_{u}}\left|u_{i}\right|$ with $\alpha$ the electric to thermal conversion factor. For simplicity, a linear relationship is assumed here but a more detailed model could be used depending on the heating system, provided it is linearized. The peak thermal cooling load of the building is $45 \mathrm{~kW}$ for the summer period. The input constraint set $\mathcal{U}$ specifies maximum and minimum cooling levels in the rooms so that $u_{i, \min } \leq u_{i} \leq u_{i, \max }=0$ for each thermal zone input, reflecting the sizing of the equipment. The state constraints $\mathcal{X}$ specifies temperature zones in the constraints so that the temperature is maintained between $20^{\circ} \mathrm{C}$ and $25^{\circ} \mathrm{C}$.

Details of the bidding are as follows: at time $t_{0}=0$, the building starts in initial condition $x_{0}$. The tracking period starts at time $t_{1}$ and ends at time $t_{2}$, therefore leaving a "preparation" period for the building controller. The capacity bid consists of the commitment of a baseline consumption during the tracking period $\mathbf{u}_{\text {nom }}$ and up-down regulation limits around that baseline for power tracking. Up-down regulation bids result in a "box" uncertainty set. We therefore fix the basic uncertainty shape as the unit box

$$
\hat{\mathcal{R}}_{\text {box }}=\left\{\boldsymbol{r} \mid\|\boldsymbol{r}\|_{\infty} \leq 1\right\}
$$

For the external disturbance from weather and internal gains, the disturbance set is defined as follows

$$
\mathcal{W}=\left\{\boldsymbol{w}_{\text {nom }}+\boldsymbol{w}_{\text {stoch }} \mid \boldsymbol{w}_{\text {stoch }, \mathrm{i}}^{T} Q_{i} \boldsymbol{w}_{\text {stoch, } \mathrm{i}} \leq 1, i=1,2,3\right\}
$$

As such, $\mathcal{W}$ is the direct product of three "uncorrelated" ellipsoidal uncertainty sets so that $\mathcal{W}=\mathcal{W}_{\text {sun }} \times \mathcal{W}_{\text {gains }} \times \mathcal{W}_{\text {temp }}$. $\boldsymbol{w}_{\text {nom }}$ is the nominal prediction of the uncertainty over the prediction horizon and $\boldsymbol{w}_{\text {stoch }}$ is the stochastic part of the uncertainty around the prediction. The three ellipsoids represent confidence sets that should cover a reasonable part of the possible outcomes for the disturbance. The choice of $Q_{i}$ determines the size of the set $\mathcal{W}$ and should be done so that $\mathcal{W}$ contains the actual weather forecast with a high confidence (see, e.g. [42]). Generally speaking, the selection of good uncertainty sets in classical robust optimization are a subject of active research [43] and fall outside the scope of the present work, but notice that rather than fixing the uncertainty $\mathcal{W}$, the method proposed in this work could also be used to optimize for $\mathcal{W}$ as well and by doing so, evaluate how much prediction error in the weather and the internal gains can be accommodated.

Finally we have $\hat{\Xi}=\hat{\mathcal{R}} \times \mathcal{W}$.

We consider here an affine controller and modifier function as in (30). Following the rules of the Swiss ancillary market, the bid is a fixed up/down capacity over the tracking period. This means the allowable modifier function is a uniform scaling of the uncertainty set (that is, time-varying capacity is not allowed). For clarity we keep the description of the uncertainty split between the reference to track and the external disturbance, so that: $\boldsymbol{\xi}=(\boldsymbol{r}, \boldsymbol{w})$ and $\boldsymbol{\nu}=\left(\boldsymbol{\nu}_{\mathcal{R}}, \boldsymbol{\nu}_{\mathcal{W}}\right)$. We assume the weather uncertainty is unknown at the time of the decision whereas the reference is revealed as it needs to be tracked: this results in an information structure that is depicted in Figure 3. We see that the modifier function could theoretically modify the uncertainty set so as to "mix" the

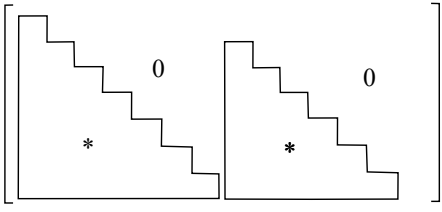

(a) $\mathcal{I}$

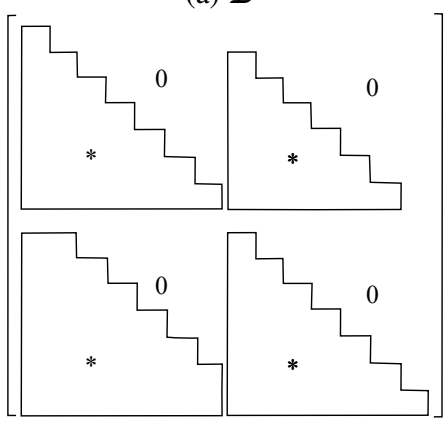

(b) $\hat{\mathcal{I}}$

Fig. 3: Information structure for the example. (a) shows that decisions at time step $t$ can depend on the reference up to time $t$ and disturbance up to time $t-1$. (b) is the resulting information structure for the modifier function.

external disturbance and the reference. In this application, it would not have physical sense so it is preferable to keep a block diagonal structure for the modifier's information structure. The disturbance uncertainty set is fixed a priori while the reference set can be modified. Furthermore, in the case that the reference set is a fixed up/down box along the horizon then the reference tracking set can only be scaled uniformly so that the modifier function will reduce to the simpler form:

$$
\mathbf{L}=\left(\begin{array}{lr}
\lambda \mathbb{I}_{N} & 0_{N, N n_{u}} \\
0_{N n_{w}, N} & \mathbb{I}_{N n_{w}}
\end{array}\right)
$$

To maximize the up/down capacity bid, it suffices to maximize the scaling factor $\lambda$. Notice that enforcing (59) implicitly enforces the requirement that $\nu \in \hat{\mathcal{I}}$. The description of the uncertainty set $\hat{\Xi}=\hat{\mathcal{R}} \times \mathcal{W}$ can easily be put under the form of equation (29) since it is the direct product of a polyhedron with three ellipsoids.

The aim of the building operator is to maximize the financial reward gained from participating in the reserve market. In accordance with the swiss market regulation, a payment proportional to the bid is made to the reserve provider, while the energy is bought at the day-ahead price, yielding the cost function $J=\mathbf{c}_{\mathbf{e}}^{T} \overline{\mathbf{u}}-c_{\text {comm }} \lambda$ where $\mathbf{c}_{\mathbf{e}}$ is the vector of timevarying prices of electricity, $\overline{\mathbf{u}}$ is the baseline consumption and $c_{\text {comm }}$ is the unit reward price of the power tracking commitment (hence promising to track $\pm 1 \mathrm{~kW}$ for the commitment period is rewarded at the price $c_{\text {comm }}$ ). Notice that according to the Swiss market rule, the deviation in power consumption resulting from the tracking still needs to be paid for, therefore adding a stochastic component to the cost. It has however been shown in [44] that this part is minor and can therefore be neglected. 

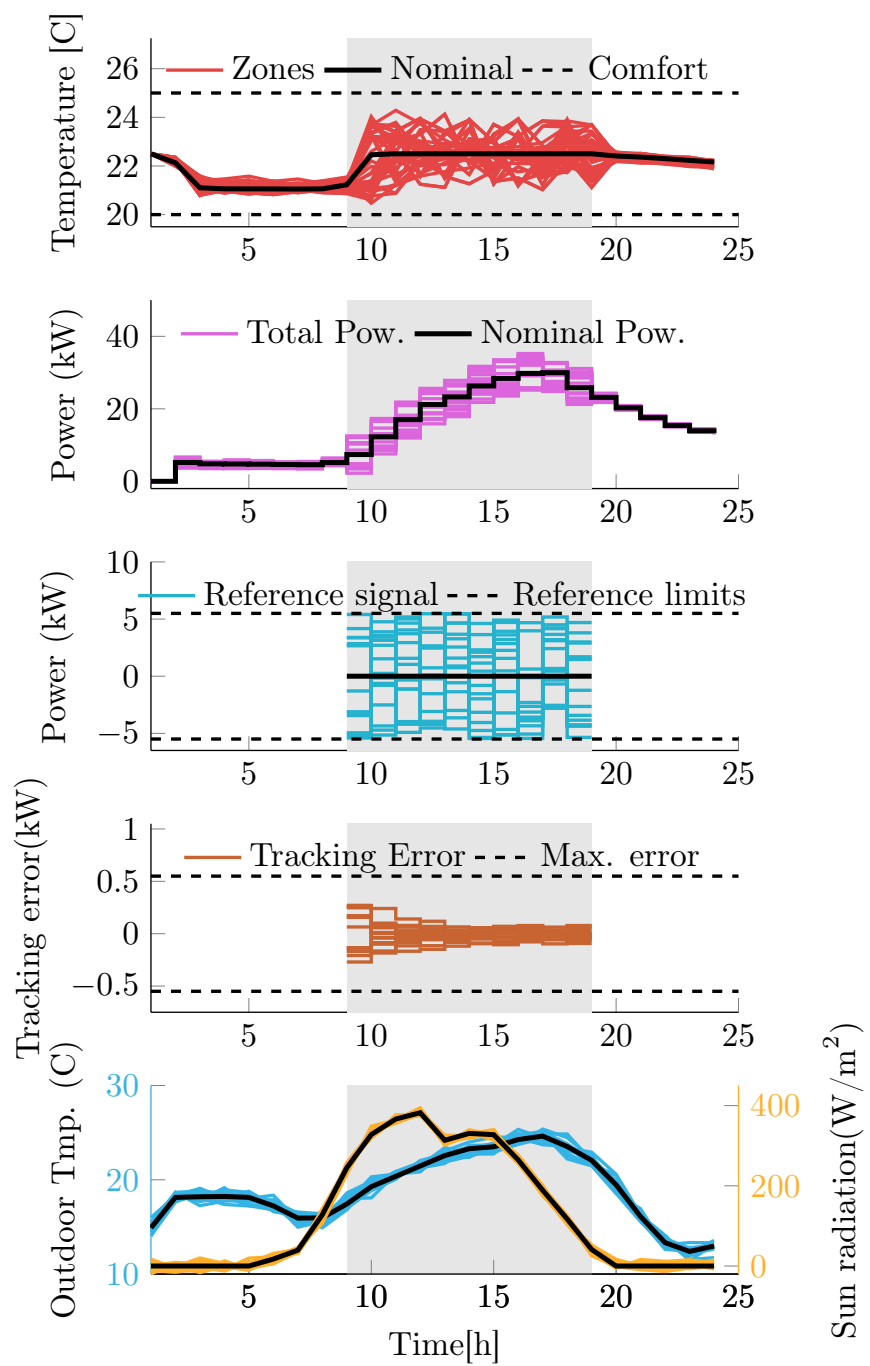

Fig. 4: Trajectories for different weather and reference scenarios in the optimized reference set. Shaded region is the tracking commitment period. Black lines show the 'nominal' scenario where the reference is zero and the weather takes its predicted value. From top to bottom: temperature in zones, total power consumption, ACS, tracking error, and weather scenarios.

The tracking error is sized proportionally to the tracking requirement so that tracking errors amounting up to $10 \%$ of the maximum tracking requirement are allowed. This yields:

$$
\mathcal{E}:=\left\{\boldsymbol{e} \mid\|\boldsymbol{e}\|_{\infty} \leqslant 0.1 \lambda\right\}
$$

which results in a tractable reformulation as suggested in section IV-C

A horizon of one day with a time step of one hour is considered. For the sake of illustration, we take $c_{e}^{t}<<c_{\text {comm }}$ in order to favor participation in the tracking commitment. The problem solved is a second-order cone problem with 200,000 non-zero variables and 900 second-order cone constraints. Solving time on a $2.7 \mathrm{GHz}$ i-Core 7 platform was 7 seconds. The optimal value of $\lambda$ is 5.4, meaning that the building can offer a $5.4 \mathrm{~kW}$ up/down power tracking capacity for a period of 10 hours. This represents $8 \%$ of the peak cooling power and $36 \%$ of the average power consumption for that day.

Figure 4 shows the trajectories generated in response to randomly generated weather and reference signals inside the reference sets. In each of the plots, the shaded band shows the reference tracking times. The different plots shows the average temperature in the building as well as in individual zones, the total power consumption in the building, the requested power consumption to be tracked on top of the nominal consumption, and the tracking error. It can be observed that in the nominal case, the power consumption increase during the day to compensate for higher solar radiation and outside temperature as shown in the bottom plot. Therefore, the baseline consumption varies during the day to maintain temperature at the nominal value of $23{ }^{\circ} \mathrm{C}$. In addition, it is seen how the temperature and power consumption changes in response to varying tracking requests (depicted in the middle plot). As a result of the requested increase or decrease of the power consumption, the temperature respectively drops or rises in the rooms, within the prescribed comfort constraints. Notice that outside the tracking period the building is only subject to uncertainty in the weather and internal gains, while during the tracking period, it has to modify its power consumption in response to the tracking signal. That causes a larger variability of the inputs and outputs during that period.

\section{B. Influence of the integral constraint in the reference set}

Frequency control bids theoretically impose the providers to be able to offer up or down regulation for long periods of time, which can be limiting for loads. In this section, we propose a way of mitigating this issue by using time-correlated (meaning the constraint describing the set couple different time stages) reference sets with integral constraints that capture more accurately the capabilities of the load. Let us consider an uncertainty set of the form

$$
\hat{\mathcal{R}}_{\text {batt }}=\left\{\mathbf{r} \mid \begin{array}{ll}
s_{0}=\bar{s}_{0} & \\
0 \leq s_{t} \leq s_{\text {max }}, & \forall t \in \mathbb{Z}_{[1, n]} \\
s_{t+1}=s_{t}+r_{t} & \forall t \in \mathbb{Z}_{[1, n]} \\
-1 \leq r_{t} \leq 1 & \forall t \in \mathbb{Z}_{[1, n]}
\end{array}\right\}
$$

This set is analogous to the feasible set of a simplified battery model where $s_{t}$ represents the state of charge of that battery. Therefore, we will refer to this uncertainty set as the "battery" reference set. Notice that contrary to the box reference set, the battery reference set is time-correlated, and our approach directly allows to account for that.

We respectively consider a box reference set (57) and a battery reference set (60) and compute the maximum bid that can be offered. Notice that the design of battery reference set requires the choice of a value for the integral constraint limit $s_{\max }$. this value was chosen as the worst case value of the integral of actual realization of the ACS over the year 2013. Therefore, for both reference sets, the system would at least be robust to any occurrence of the ACS that happened in 2013. The value computed is given by $s_{\max }=5.6$. This suggests that the tracking signal is relatively well-behaved in the sense 


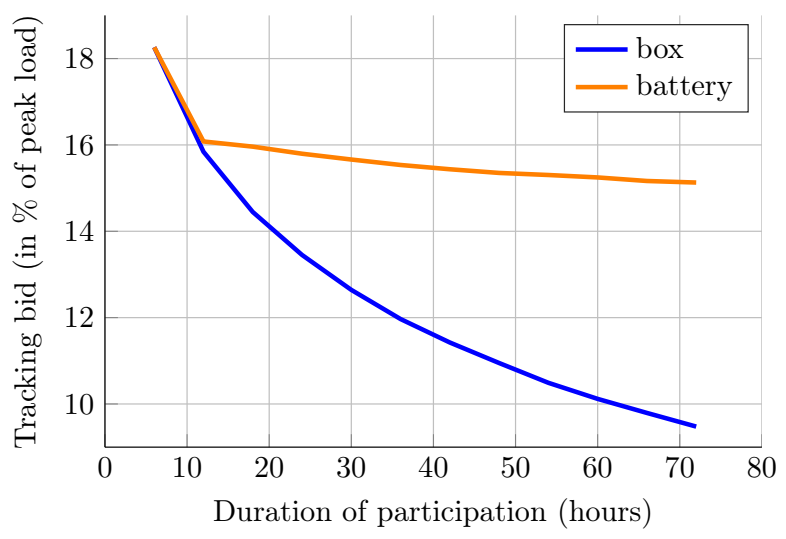

Fig. 5: Tracking capacity bid versus duration of participation for box and battery reference sets.

that it does not typically ask for maximum positive or negative power tracking for long periods of time.

To study the influence of the integral limit in the reference set, the tracking bid is evaluated as a function of the duration of the tracking commitment. the preparation time is kept at 8 hours. The weather is considered known perfectly in advance in this case to rule out other factors of uncertainty in the computation. Beyond 66 hours, the computational burden becomes prohibitive. The maximum bids for the battery and the box uncertainty sets are reported on Figure 5. We can observe that, beyond $12 \mathrm{~h}$ of consecutive participation, introducing an integral limit for the tracking commitment allows to increase the tracking bid, and more so as the duration of participation time increases. Thanks to the integral constraints, situations of long lasting positive or negative tracking requests are ruled out, thus relieving the tracking requirements on the building, and leading to less conservative solutions.

\section{CONCLUSION}

In this paper, we have formulated the problem of optimal robust tracking commitment and proposed a computationally tractable solution method. By implicit modification of uncertainty sets, the set of possible reference trajectories that can be tracked under additive disturbance with a guaranteed error bound can be efficiently computed over a finite prediction horizon. The presented tracking commitment framework is representative of many practical problems encountered in the hierarchical control of complex systems, that requires communication of tracking capability of subsystems to an upper level control layer. We have illustrated the description capability of the framework and the solution method with a practical example that investigates ancillary service provision to the power grid by a commercial building.

\section{APPENDIX A}

\section{POLYTOPIC DESCRIPTION OF THE FEASIBILITY SET $\mathcal{Q}$}

The dense form of the system equations (1), which describes the evolution of the system for $N$ steps, is given by

$$
\begin{aligned}
& \boldsymbol{x}=\mathbf{A} x_{0}+\mathbf{B} \boldsymbol{u}+\mathbf{E} \boldsymbol{\omega} \\
& \boldsymbol{y}=\mathbf{C} \boldsymbol{x}+\mathbf{D} \boldsymbol{u}
\end{aligned}
$$

The matrices $\mathbf{A} \in \mathbb{R}^{(N+1) n_{x} \times n_{x}}, \mathbf{B} \in \mathbb{R}^{(N+1) n_{x} \times N n_{u}}, \mathbf{E} \in$ $\mathbb{R}^{(N+1) n_{x} \times N n_{x}}, \mathbf{C} \in \mathbb{R}^{N n_{y} \times(N+1) n_{x}}$ and $\mathbf{D} \in \mathbb{R}^{N n_{y} \times N n_{u}}$ are defined as

$$
\begin{gathered}
\mathbf{A}:=\left[\begin{array}{c}
I_{n_{x}} \\
A \\
A^{2} \\
\vdots \\
A^{N}
\end{array}\right], \mathbf{E}:=\left[\begin{array}{cccc}
0 & \cdots & \cdots & 0 \\
I_{n_{x}} & 0 & \cdots & \vdots \\
A & I_{n_{x}} & \cdots & \vdots \\
\vdots & \vdots & \ddots & \vdots \\
A^{N-1} & A^{N-2} & \cdots & I_{n_{x}}
\end{array}\right] \\
\mathbf{B}:=\mathbf{E}\left(I_{N} \otimes B\right), \mathbf{C}:=\left[\begin{array}{ll}
I_{N} \otimes C & \mathbf{0}
\end{array}\right], \mathbf{D}:=I_{N} \otimes D
\end{gathered}
$$

The polytopic state, input constraints and the disturbance set can be described as

$$
\begin{aligned}
\mathcal{X} & :=\left\{\boldsymbol{\phi} \in \mathbb{R}^{N n_{x}}: \mathbf{F}_{x} \boldsymbol{\phi} \leqslant \mathbf{f}_{x}\right\} \\
\mathcal{U} & :=\left\{\boldsymbol{u} \in \mathbb{R}^{N n_{u}}: \mathbf{F}_{u} \boldsymbol{u} \leqslant \mathbf{f}_{u}\right\} \\
\mathcal{W} & :=\left\{\boldsymbol{w} \in \mathbb{R}^{N n_{x}}: \mathbf{F}_{w} \boldsymbol{w} \leqslant \mathbf{f}_{w}\right\}
\end{aligned}
$$

The matrices used in the descriptions (3) and (45) of the feasibility set $\mathcal{Q}$ are given by

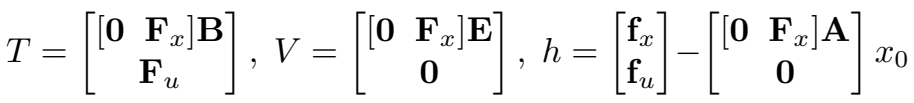

$$
\begin{aligned}
& P:=\mathbf{C B}+\mathbf{D}, \quad S=\mathbf{C E}, \quad \overline{\boldsymbol{y}}=\mathbf{C A} x_{0} \\
& H:=\left[\begin{array}{c}
T \\
-P
\end{array}\right], \quad Q=\left[\begin{array}{cc}
\mathbf{0} & V \\
-S & G
\end{array}\right], \quad q=\left[\begin{array}{c}
h \\
g+\overline{\boldsymbol{y}}
\end{array}\right]
\end{aligned}
$$

where 0's are matrices of zeros with proper dimensions.

\section{APPENDIX B}

\section{PROOFS FOR THEOREMS OF SECTION II-B}

Notations: Given a set of indices $\mathcal{J}$, let $\overline{\mathcal{J}}$ be the complementary of $\mathcal{J}$ in $\mathbb{Z}_{[1, n]}$. Denote $m$ the cardinality of $\mathcal{J}$. As $x_{\mathcal{J}}$ denotes the entries of $x$ indexed by $\mathcal{J}, \boldsymbol{\nu}_{\mathcal{J}}$ denotes the function from $\mathbb{R}^{n}$ into $\mathbb{R}^{m}$ formed by the outputs of $\nu$ indexed by $\mathcal{J}$. Given $\mathcal{J}$, we also overload notations and denote $\boldsymbol{\nu}\left(x_{\mathcal{J}}, x_{\overline{\mathcal{J}}}\right)$ to make explicit the respective dependency of $\boldsymbol{\nu}$ on $x_{\mathcal{J}}$ and $x_{\bar{J}}$. Accordingly, denote $\boldsymbol{\nu}\left(x_{\mathcal{J}},.\right)$ the restriction of $\boldsymbol{\nu}$ to $\left\{x_{\mathcal{J}}\right\} \times \mathbb{R}^{n-m}$.

Proof of Lemma 3. Consider two information structures $\mathcal{I}_{1}$ and $\mathcal{I}_{2}$. Suppose $f \in \mathcal{F}\left(\mathcal{I}_{1}\right), \mathcal{F}\left(\mathcal{I}_{2}\right)$. Let $x, x^{\prime}$ be such that $x_{\mathcal{I}_{1} \cap \mathcal{I}_{2}}=x_{\mathcal{I}_{1} \cap \mathcal{I}_{2}}^{\prime}$. Choose $y$ such that $y_{\mathcal{I}_{1}}=x_{\mathcal{I}_{1}}$ and $y_{\mathcal{I}_{2}}=x_{\mathcal{I}_{2}}^{\prime}$ (this is possible because $x_{\mathcal{I}_{1} \cap \mathcal{I}_{2}}=x_{\mathcal{I}_{1} \cap \mathcal{I}_{2}}^{\prime}$ ). Since $f \in \mathcal{F}\left(\mathcal{I}_{1}\right)$, we have that $f(x)=f(y)$. Similarly, $f \in \mathcal{F}\left(\mathcal{I}_{2}\right)$ implies that $f\left(x^{\prime}\right)=f(y)$. Together this gives $f(x)=f(y)=f\left(x^{\prime}\right)$ for all $x, x^{\prime}$ such that $x_{\mathcal{I}_{1} \cap \mathcal{I}_{2}}=x_{\mathcal{I}_{1} \cap \mathcal{I}_{2}}^{\prime}$ i.e. $f \in \mathcal{F}\left(\mathcal{I}_{1} \cap \mathcal{I}_{2}\right)$. Noticing that $\bigcap_{k} \mathcal{I}_{k}=\mathcal{I}_{1} \cap\left(\bigcap_{k \neq 1} \mathcal{I}_{k}\right)$, it is straightforward to extend the argument above to the intersection of finitely many information structures.

Proof of Lemma 4. By convention, $\hat{\mathcal{I}}_{k}=\mathbb{Z}_{[1, n]}$ if $\left\{i \mid k \in \mathcal{I}_{i}\right\}$ is empty.

Direction $\Longleftarrow:$ Assume $\boldsymbol{g} \in \mathcal{F}(\hat{\mathcal{I}})$. Consider $(x, \hat{x})$ such that $x_{\mathcal{I}_{j}}=x_{\mathcal{I}_{j}}^{\prime}$ and $\boldsymbol{f} \in \mathcal{F}(\mathcal{I})$. Let us prove that $\boldsymbol{f} \circ \boldsymbol{g}(x)=$ $\boldsymbol{f} \circ \boldsymbol{g}\left(x^{\prime}\right)$. Let us denote $y=\boldsymbol{g}(x)$ and $y^{\prime}=\boldsymbol{g}\left(x^{\prime}\right)$. Let us consider any $k \in \mathcal{I}_{j}$. Then according to equation (21), we have $\hat{\mathcal{I}}_{k} \subseteq \mathcal{I}_{j}$ and hence $x_{\hat{\mathcal{I}}_{k}}=x_{\hat{\mathcal{I}}_{k}}^{\prime}$. In turn this implies $y_{k}=y_{k}^{\prime}$ by definition of $\mathcal{F}(\hat{\mathcal{I}})$. Since this holds for all $k \in \mathcal{I}_{j}$, it holds that 
$y_{\mathcal{I}_{j}}=y_{\mathcal{I}_{j}}^{\prime}$ and therefore $\boldsymbol{f} \circ \boldsymbol{g}(x)=\boldsymbol{f}(y)=\boldsymbol{f}\left(y^{\prime}\right)=\boldsymbol{f} \circ \boldsymbol{g}\left(x^{\prime}\right)$ since $f \in \mathcal{F}(\mathcal{I})$.

Direction $\Longrightarrow$ : Assume $\boldsymbol{g} \notin \mathcal{F}(\hat{\mathcal{I}})$. There exists an index $j$ such that $g_{j} \notin \mathcal{F}\left(\hat{\mathcal{I}}_{j}\right)$. Since $\hat{\mathcal{I}}_{j}=\bigcap_{\left\{i \mid j \in \mathcal{I}_{i}\right\}} \mathcal{I}_{i}$ we can use Lemma 3 to conclude that there exists $i$ such that $g_{j} \notin \mathcal{F}\left(\mathcal{I}_{i}\right)$ and $j \in \mathcal{I}_{i}$. (The intersection is non-empty since if it was then $\hat{\mathcal{I}}_{j}=\mathbb{Z}_{[1, n]}$, which contradicts the possibility that $g_{j}(x) \neq$ $\left.g_{j}\left(x^{\prime}\right)\right)$. Then there exist $x$ and $x^{\prime}$ such that $x_{\mathcal{I}_{i}}=x_{\mathcal{I}_{i}}^{\prime}$ and $g_{j}(x) \neq g_{j}\left(x^{\prime}\right)$. Consider the function $\boldsymbol{f}$ defined as follows: $\forall k \neq i, f_{k}$ is identically 0 . This trivially implies $f_{k} \in \mathcal{F}\left(\mathcal{I}_{k}\right)$ no matter what $\mathcal{I}$ is. Define $f_{i}$ as:

$$
\left\{\begin{array}{l}
f_{i}(y)=1 \text { if } y_{j}=g_{j}\left(x^{\prime}\right) \\
f_{i}(y)=0 \text { otherwise }
\end{array}\right.
$$

Consider $y, y^{\prime}$ such that $y_{\mathcal{I}_{i}}=y_{\mathcal{I}_{i}}^{\prime}$. Since $j \in \mathcal{I}_{i}$, we have $y_{j}=y_{j}^{\prime}$ and hence $f_{i}(y)=f_{i}\left(y^{\prime}\right)$. Therefore $f_{i} \in \mathcal{F}\left(\mathcal{I}_{i}\right)$ and $\boldsymbol{f} \in \mathcal{F}(\mathcal{I})$. However, $f_{i} \circ \boldsymbol{g}(x)=0$ and $f_{i} \circ \boldsymbol{g}\left(x^{\prime}\right)=1$ by definition of $f_{i}$. Putting everything together, we can conclude that $x_{\mathcal{I}_{i}}=x_{\mathcal{I}_{i}}^{\prime}$ and $\boldsymbol{f} \circ \boldsymbol{g}(x) \neq \boldsymbol{f} \circ \boldsymbol{g}\left(x^{\prime}\right)$, therefore $\boldsymbol{f} \circ \boldsymbol{g} \notin$ $\mathcal{F}(\mathcal{I})$.

Proof of Lemma $5 \mathcal{G} \subseteq \mathcal{F}(\mathcal{I})$ : It directly follows from Lemma 4

$\mathcal{F}(\mathcal{I}) \subseteq \mathcal{G}$ : Consider $\boldsymbol{g} \in \mathcal{F}(\mathcal{I})$. Showing that there exists $f \in \mathcal{F}(\mathcal{I})$ such that $\boldsymbol{g}=\boldsymbol{f} \circ \boldsymbol{\nu}$ is equivalent to showing that $\boldsymbol{f}=\boldsymbol{g} \circ \boldsymbol{\nu}^{-1} \in \mathcal{F}(\mathcal{I})$ ( $\boldsymbol{\nu}$ is a bijection). It is done by contradiction. Suppose $f \notin \mathcal{F}(\mathcal{I})$. This means that for some $k, f_{k} \notin \mathcal{F}\left(\mathcal{I}_{k}\right)$. To lighten notation, let $\mathcal{I}_{k}=\mathcal{J}$. There exist $y, y^{\prime}$ such that $y_{\mathcal{J}}=y_{\mathcal{J}}^{\prime}$ and $f_{k}(y) \neq f_{k}\left(y^{\prime}\right)$. By definition of $\hat{\mathcal{I}}, \boldsymbol{\nu}_{\mathcal{J}}$ cannot depend on elements of $\overline{\mathcal{J}}$, i.e. $x_{\mathcal{J}}=x_{\mathcal{J}}^{\prime} \Longrightarrow$ $\boldsymbol{\nu}_{\mathcal{J}}(x)=\boldsymbol{\nu}_{\mathcal{J}}\left(x^{\prime}\right)$. Fix $x \in \mathbb{R}^{n}$. We divide the remainder of the proof in intermediate steps for clarity.

Bijectivity of $\boldsymbol{\nu}_{\mathcal{J}}\left(., x_{\overline{\mathcal{J}}}\right)$ : Notice that $\boldsymbol{\nu}_{\overline{\mathcal{J}}}\left(x_{\mathcal{J}},.\right)$ is injective in $\mathbb{R}^{n-m}$ since $\boldsymbol{\nu}$ is injective. Denoting $V\left(x_{\mathcal{J}}\right)=$ $\boldsymbol{\nu}_{\overline{\mathcal{J}}}\left(x_{\mathcal{J}}, \mathbb{R}^{n-m}\right)$, by continuity of $\boldsymbol{\nu}, V\left(x_{\mathcal{J}}\right)$ is an open set. By injectivity of $\boldsymbol{\nu}$, if $\boldsymbol{\nu}_{\mathcal{J}}(x)=\boldsymbol{\nu}_{\mathcal{J}}\left(x^{\prime}\right)$ with $x_{\mathcal{J}} \neq x_{\mathcal{J}}^{\prime}$, then $V(x)$ and $V\left(x^{\prime}\right)$ are disjoint. By surjectivity of $\boldsymbol{\nu}$, it also holds that $\cup_{\left\{x_{\mathcal{J}}^{\prime} \mid \boldsymbol{\nu}_{\mathcal{J}}\left(x_{\mathcal{J}}, x_{\overline{\mathcal{J}}}\right)=\boldsymbol{\nu}_{\mathcal{J}}\left(x_{\mathcal{J}}^{\prime}, x_{\bar{J}}\right)\right\}} V\left(x_{\mathcal{J}}^{\prime}\right)=\mathbb{R}^{n-m}$. Since $\mathbb{R}^{n-m}$ is connected, it cannot be covered by a non-trivial union of disjoint open sets, which implies that $\left\{x_{\mathcal{J}}^{\prime} \mid \boldsymbol{\nu}_{\mathcal{J}}\left(x_{\mathcal{J}}, x_{\overline{\mathcal{J}}}\right)=\right.$ $\left.\boldsymbol{\nu}_{\mathcal{J}}\left(x_{\mathcal{J}}^{\prime}, x_{\bar{J}}\right)\right\}$ is reduced to $\left\{x_{\mathcal{J}}\right\}$, which in other words means injectivity of $\boldsymbol{\nu}_{\mathcal{J}}\left(., x_{\overline{\mathcal{J}}}\right)$.

Surjectivity of $\boldsymbol{\nu}_{\mathcal{J}}\left(., x_{\overline{\mathcal{J}}}\right)$ directly follows from the surjectivity of $\boldsymbol{\nu}$. Indeed, $\forall y \in \mathbb{R}^{m}$ there exist $x^{\prime}$ such that $\boldsymbol{\nu}_{\mathcal{J}}\left(x^{\prime}\right)=y$. Then $\boldsymbol{\nu}_{\mathcal{J}}\left(x_{\mathcal{J}}^{\prime}, x_{\overline{\mathcal{J}}}\right)=y$. Together, this proves the bijectivity of $\boldsymbol{\nu}_{\mathcal{J}}\left(., x_{\overline{\mathcal{J}}}\right)$ for all $x_{\overline{\mathcal{J}}}$.

Bijectivity of $\boldsymbol{\nu}_{\overline{\mathcal{J}}}\left(x_{\mathcal{J}},.\right)$ : Injectivity directly follows from the injectivity of $\nu$. For $x_{\mathcal{J}}$ fixed, by injectivity of $\boldsymbol{\nu}_{\mathcal{J}}\left(., x_{\bar{J}}\right)$ there does not exist any other $x_{\mathcal{J}}^{\prime}$ such that $\boldsymbol{\nu}_{\mathcal{J}}\left(x_{\mathcal{J}}, x_{\overline{\mathcal{J}}}\right)=\boldsymbol{\nu}_{\mathcal{J}}\left(x_{\mathcal{J}}^{\prime}, x_{\overline{\mathcal{J}}}\right)$. Therefore, surjectivity of $\boldsymbol{\nu}$ implies that $\boldsymbol{\nu}_{\overline{\mathcal{J}}}\left(x_{\mathcal{J}}, \mathbb{R}^{n-m}\right)=\mathbb{R}^{n-m}$, i.e. surjectivity of $\boldsymbol{\nu}_{\overline{\mathcal{J}}}\left(x_{\mathcal{J}},.\right)$.

Contradiction : Consider $x_{\mathcal{J}}$ such that $\boldsymbol{\nu}_{\mathcal{J}}\left(x_{\mathcal{J}}, x_{\overline{\mathcal{J}}}\right)=y_{\mathcal{J}}$. Bijectivity of $\boldsymbol{\nu}_{\mathcal{J}}\left(., x_{\overline{\mathcal{J}}}\right)$ ensures its existence. In turn, bijectivity of $\boldsymbol{\nu}_{\overline{\mathcal{J}}}\left(x_{\mathcal{J}},.\right)$ ensures that there exists $x_{\overline{\mathcal{J}}}, x_{\overline{\mathcal{J}}}^{\prime}$ such that $\boldsymbol{\nu}_{\overline{\mathcal{J}}}\left(x_{\mathcal{J}}, x_{\overline{\mathcal{J}}}\right)=y_{\overline{\mathcal{J}}}$ and $\boldsymbol{\nu}_{\overline{\mathcal{J}}}\left(x_{\mathcal{J}}, x_{\overline{\mathcal{J}}}^{\prime}\right)=y_{\overline{\mathcal{J}}}^{\prime}$. Combining the results above gives $\boldsymbol{\nu}\left(x_{\mathcal{J}}, x_{\overline{\mathcal{J}}}\right)=y$ and $\boldsymbol{\nu}\left(x_{\mathcal{J}}, x_{\overline{\mathcal{J}}}^{\prime}\right)=y^{\prime}$. Then, $g_{k}(x)=f_{k} \circ \boldsymbol{\nu}(x)=f_{k}(y)$ and similarly $g_{k}\left(x^{\prime}\right)=$ $f_{k} \circ \boldsymbol{\nu}\left(x^{\prime}\right)=f_{k}\left(y^{\prime}\right)$. Finally, this shows that $g_{k}(x) \neq g_{k}\left(x^{\prime}\right)$ which implies $g_{k} \notin \mathcal{F}\left(\mathcal{I}_{k}\right)$. this contradicts the assumption that $g \in \mathcal{F}(\mathcal{I})$. Finally, this confirms that $f \in \mathcal{F}(\mathcal{I})$.

\section{ACKNOWLEDGMENT}

The research leading to these results has received funding from the European Research Council under the European Union's Seventh Framework Programme (FP/2007-2013) / ERC Grant Agreement n. 307608: BuildNet and the Swiss National Science Foundation under the GEMS project (Green Energy Management of Structures, grant number 200021 137985). The authors would like to thank Swissgrid Ltd. for providing data and Giorgos Stathopoulos for fruitful discussions.

\section{REFERENCES}

[1] Y. Rebours, D. Kirschen, M. Trotignon, and S. Rossignol, "A Survey of Frequency and Voltage Control Ancillary Services mdash;Part I: Technical Features," IEEE Transactions on Power Systems, vol. 22, no. 1, pp. 350-357, Feb. 2007.

[2] T. Gorecki, A. Bitlislioğlu, G. Stathopoulos, and C. N. Jones, "Guaranteeing input tracking for constrained systems: theory and application to demand response," in the 2015 American Control Conf. (ACC), 2015.

[3] M. Maasoumy, C. Rosenberg, A. Sangiovanni-Vincentelli, and D. Callaway, "Model predictive control approach to online computation of demand-side flexibility of commercial buildings HVAC systems for Supply Following," in American Control Conf. (ACC), 2014, Jun. 2014, pp. $1082-1089$.

[4] S. Raković, "Invention of Prediction Structures and Categorization of Robust MPC Syntheses," in Proc. 4th IFAC Nonlinear Model Predictive Control Conf., M. Lazar, Ed., Aug. 2012, pp. 245-273.

[5] D. Mayne, M. Seron, and S. Raković, "Robust model predictive control of constrained linear systems with bounded disturbances," Automatica, vol. 41, no. 2, pp. 219-224, Feb. 2005.

[6] S. V. Raković, B. Kouvaritakis, R. Findeisen, and M. Cannon, "Homothetic tube model predictive control," Automatica, vol. 48, no. 8, pp. 1631-1638, Aug. 2012.

[7] S. Raković, B. Kouvaritakis, M. Cannon, C. Panos, and R. Findeisen, "Parameterized Tube Model Predictive Control," IEEE Transactions on Automatic Control, vol. 57, no. 11, pp. 2746-2761, Nov. 2012.

[8] P. J. Goulart, E. C. Kerrigan, and J. M. Maciejowski, "Optimization over state feedback policies for robust control with constraints," Automatica, vol. 42, no. 4, pp. 523-533, Apr. 2006.

[9] A. Ben-Tal, L. El Ghaoui, and A. Nemirovski, Robust Optimization. Princeton University Press, 2009

[10] A. Lorca and X. Sun, "Adaptive Robust Optimization With Dynamic Uncertainty Sets for Multi-Period Economic Dispatch Under Significant Wind," IEEE Transactions on Power Systems, vol. 30, no. 4, pp. 17021713, Jul. 2015

[11] A. Georghiou, W. Wiesemann, and D. Kuhn, "Generalized decision rule approximations for stochastic programming via liftings," Mathematical Programming, pp. 1-38, May 2014.

[12] J. Warrington, P. Goulart, S. Mariethoz, and M. Morari, "Policy-Based Reserves for Power Systems," IEEE Transactions on Power Systems, vol. 28, no. 4, pp. 4427-4437, Nov. 2013.

[13] F. Oldewurtel, T. Borsche, M. Bucher, P. Fortenbacher, M. G. V. T. Haring, J. L. Mathieu, O. Megel, E. Vrettos, and G. Andersson, "A framework for and assessment of demand response and energy storage in power systems," in Bulk Power System Dynamics and Control - IX Optimization, Security and Control of the Emerging Power Grid (IREP), 2013 IREP Symposium, 2013, pp. 1-24.

[14] E. Vrettos, F. Oldewurtel, G. Andersson, and F. Zhu, "Robust Provision of Frequency Reserves by Office Building Aggregations," in Proceedings of the 19th IFAC World Congress, Cape Town, South Africa, 2014.

[15] M. Balandat, F. Oldewurtel, M. Chen, and C. Tomlin, "Contract design for frequency regulation by aggregations of commercial buildings," in 2014 52nd Annual Allerton Conf. on Communication, Control, and Computing (Allerton), Sep. 2014, pp. 38-45. 
[16] X. Zhang, M. Kamgarpour, P. Goulart, and J. Lygeros, "Selling robustness margins: A framework for optimizing reserve capacities for linear systems," in 2014 IEEE 53rd Annual Conf. on Decision and Control (CDC), Dec. 2014, pp. 6419-6424.

[17] E. Vrettos, F. Oldewurtel, and G. Andersson, "Robust EnergyConstrained Frequency Reserves From Aggregations of Commercial Buildings," IEEE Transactions on Power Systems, vol. PP, no. 99, pp. $1-14,2016$.

[18] H. Hao, B. Sanandaji, K. Poolla, and T. Vincent, "Aggregate Flexibility of Thermostatically Controlled Loads," IEEE Transactions on Power Systems, vol. Early Access Online, 2014.

[19] L. Zhao and W. Zhang, "A geometric approach to virtual battery modeling of thermostatically controlled loads," in 2016 American Control Conference (ACC), Jul. 2016, pp. 1452-1457.

[20] A. Nayyar, M. Negrete-Pincetic, K. Poolla, and P. Varaiya, "Durationdifferentiated energy services with a continuum of loads," in 53rd IEEE Conference on Decision and Control, Dec. 2014, pp. 1714-1719.

[21] B. Francis, "The Linear Multivariable Regulator Problem," SIAM Journal on Control and Optimization, vol. 15, no. 3, pp. 486-505, May 1977.

[22] U. Maeder and M. Morari, "Offset-free reference tracking with model predictive control," Automatica, vol. 46, no. 9, pp. 1469-1476, Sep. 2010.

[23] D. Limon, I. Alvarado, T. Alamo, and E. F. Camacho, "Robust tubebased MPC for tracking of constrained linear systems with additive disturbances," Journal of Process Control, vol. 20, no. 3, pp. 248-260, Mar. 2010.

[24] P. Falugi and D. Mayne, "Model predictive control for tracking random references," in European Control Conf. (ECC), 2013 European, Jul. 2013, pp. $518-523$.

[25] S. Di Cairano and F. Borrelli, "Constrained tracking with guaranteed error bounds," in 2013 IEEE 52nd Annual Conf. on Decision and Control (CDC), Dec. 2013, pp. 3800-3805.

[26] F. Guerra Vázquez, J. J. Rückmann, O. Stein, and G. Still, "Generalized semi-infinite programming: A tutorial," Journal of Computational and Applied Mathematics, vol. 217, no. 2, pp. 394-419, Aug. 2008.

[27] G. Still, "Solving generalized semi-infinite programs by reduction to simpler problems," Optimization, vol. 53, no. 1, pp. 19-38, Feb. 2004.

[28] A. Mitsos and A. Tsoukalas, "Global optimization of generalized semiinfinite programs via restriction of the right hand side," Journal of Global Optimization, vol. 61, no. 1, pp. 1-17, Jan. 2014.

[29] S. M. Harwood and P. I. Barton, "Lower level duality and the global solution of generalized semi-infinite programs," Optimization, vol. 65, no. 6, pp. 1129-1149, Jun. 2016.

[30] M. Diehl, B. Houska, O. Stein, and P. Steuermann, "A lifting method for generalized semi-infinite programs based on lower level Wolfe duality," Computational Optimization and Applications, vol. 54, no. 1, pp. 189210, Jun. 2012.

[31] S. Raković, E. Kerrigan, D. Mayne, and J. Lygeros, "Reachability analysis of discrete-time systems with disturbances," IEEE Transactions on Automatic Control, vol. 51, no. 4, pp. 546-561, Apr. 2006.

[32] S. V. Raković, E. C. Kerrigan, D. Q. Mayne, and K. I. Kouramas, "Optimized robust control invariance for linear discrete-time systems: Theoretical foundations," Automatica, vol. 43, no. 5, pp. 831-841, May 2007.

[33] B. L. Gorissen, I. Yanikoğlu, and D. den Hertog, "A practical guide to robust optimization," Omega, vol. 53, pp. 124-137, Jun. 2015.

[34] D. Bertsimas and A. Georghiou, "Design of Near Optimal Decision Rules in Multistage Adaptive Mixed-Integer Optimization," Operations Research, vol. 63, no. 3, pp. 610-627, Apr. 2015.

[35] J. Zhen and D. den Hertog, "Computing the Maximum Volume Inscribed Ellipsoid of a Polytopic Projection," CentER Discussion Paper Series, vol. 2015-004, Jan. 2015.

[36] Swissgrid, "Test for secondary control capability." [Online]. Available: https://www.swissgrid.ch/dam/swissgrid/experts/ancillary_services/ prequalification/D130422_Test-for-secondary-control-capability_ V2R1_EN.pdf

[37] A. Shapiro, D. Dentcheva, and A. P. Ruszczyński, Lectures on Stochastic Programming: Modeling and Theory. Society for Industrial and Applied Mathematics, Philadelphia, 2009.

[38] S. Boyd, "Distributed Optimization and Statistical Learning via the Alternating Direction Method of Multipliers," Foundations and Trends in Machine Learning, vol. 3, no. 1, pp. 1-122, 2010.

[39] N. Michael, J. Fink, and V. Kumar, "Cooperative manipulation and transportation with aerial robots," Autonomous Robots, vol. 30, no. 1, pp. 73-86, Jan. 2011.
[40] Swissgrid, "Overview of ancillary service products." [Online]. Available: https://www.swissgrid.ch/dam/swissgrid/experts/ancillary_ services/Dokumente/D100412_AS-concept_V1R0_en.pdf

[41] T. Gorecki, F. Qureshi, and C. Jones, "OpenBuild : An integrated simulation environment for building control," in 2015 IEEE Conference on Control Applications (CCA), Sep. 2015, pp. 1522-1527.

[42] K. Margellos, P. Goulart, and J. Lygeros, "On the Road Between Robust Optimization and the Scenario Approach for Chance Constrained Optimization Problems," IEEE Transactions on Automatic Control, vol. 59, no. 8, pp. 2258-2263, Aug. 2014.

[43] D. Bertsimas, V. Gupta, and N. Kallus, "Data-driven robust optimization," ArXiv e-prints, Dec. 2014.

[44] I. Lymperopoulos, F. A. Qureshi, T. Nghiem, A. A. Khatir, and C. N. Jones, "Providing ancillary service with commercial buildings: the swiss perspective," IFAC-PapersOnLine, vol. 48, no. 8, pp. 6 - 13, 2015, 9th (IFAC) Symposium on Advanced Control of Chemical Processes (ADCHEM), June 2015.

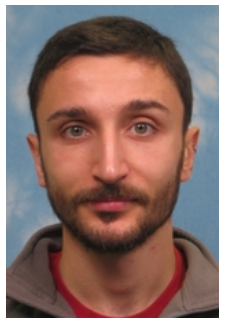

Altuğ Bitlislioğlu received his diploma in Mechanical Engineering in 2011 from the Middle Eastern Technical University (METU), Ankara, Turkey. He then received his MSc. degree in Robotics, Systems and Control in 2013 from the Eidgenössische Technische Hochschule Zürich (ETHZ). In October 2013 , he joined the group of Prof. Colin Jones in Laboratoire d'Automatique at École Polytechnique Fédérale de Lausanne (EPFL), as a PhD student. His research interests include assessment of tracking capabilities of distributed systems and optimal control methods for smart grid applications.

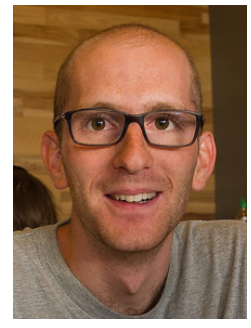

Tomasz T. Gorecki received his master degree in mechanical engineering with a minor in automatic control in 2012. He completed as master project a research project at the ONERA (French Aerospace public research Institute), studying application of model predictive control strategies for drones for area exploration. In September 2012 he joined the group of Colin Jones at the Automatic Control Laboratory at EPFL, Lausanne as a PhD student. $\mathrm{He}$ is employed in relation with the project entitled "Green energy management of structures" focusing on application of Model Predictive Control to building and smart grid applications. His interests include robust and stochastic optimization, economic MPC, invariant set theory and computation, economics of the smart grid.

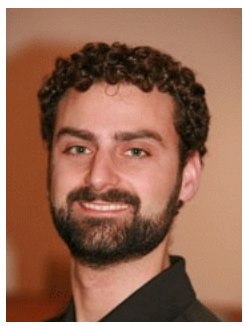

Colin N. Jones received the Bachelor's degree in Electrical Engineering and the Master's degree in Mathematics from the University of British Columbia, Vancouver, BC, Canada, and the Ph.D. degree from the University of Cambridge, Cambridge, U.K., in 2005. He is an Assistant Professor in the Automatic Control Laboratory at the École Polytechnique Fédérale de Lausanne (EPFL), Lausanne, Switzerland. He was a Senior Researcher at the Automatic Control Laboratory of the Swiss Federal Institute of Technology Zurich until 2010. His current research interests are in the areas of high-speed predictive control and optimisation, as well as green energy generation, distribution and management. 